\title{
[5C + 1S] Annulation: A Facile and Efficient Synthetic Route toward Functionalized 2,3-Dihydrothiopyran-4-ones
}

Xihe Bi, Dewen Dong*, Yan Li, Qun Liu* and Qian Zhang

Department of Chemistry, Northeast Normal University, Changchun, 130024, P. R. China

E-mail: dongdw663@nenu.edu.cn

\section{Supporting Information}

Table of Contents............................................ 1

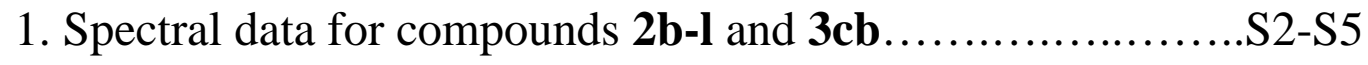

2. Copies of NMR spectra for $\mathbf{2 a - 1} \ldots \ldots \ldots \ldots \ldots \ldots \ldots \ldots \ldots \ldots . . .56-S 24$

3. Copies of NMR spectra for 3ca and 3cb.............S25-S28

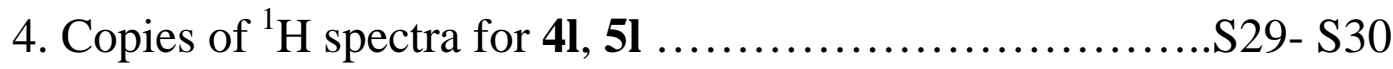


2-(Benzo[d][1,3]dioxol-5-yl)-5-benzoyl-6-ethylthio-2,3-dihydrothiopyran-4-one

(2b): Yellowish semisolid; ${ }^{1} \mathrm{H}$ NMR $\left(500 \mathrm{MHz}, \mathrm{CDCl}_{3}\right) \delta=1.26(\mathrm{t}, J=7.5 \mathrm{~Hz}, 3 \mathrm{H})$, 2.93-3.04 (m, 3H), $3.19(\mathrm{dd}, J=14.0,16.5 \mathrm{~Hz}, 1 \mathrm{H}), 4.74(\mathrm{dd}, J=3.5,14.0 \mathrm{~Hz}, 1 \mathrm{H})$, $6.02(\mathrm{~s}, 2 \mathrm{H}), 6.84(\mathrm{~d}, J=8.0 \mathrm{~Hz}, 1 \mathrm{H}), 6.90(\mathrm{~d}, J=8.0 \mathrm{~Hz}, 1 \mathrm{H}), 6.92(\mathrm{~s}, 1 \mathrm{H}), 7.44-7.47$ $(\mathrm{m}, 2 \mathrm{H}), 7.55-7.58(\mathrm{~m}, 1 \mathrm{H}), 7.88(\mathrm{~d}, J=7.5 \mathrm{~Hz}, 2 \mathrm{H}) .{ }^{13} \mathrm{C} \mathrm{NMR}\left(125 \mathrm{MHz}, \mathrm{CDCl}_{3}\right)$ $\delta=194.66,190.82,160.48,148.15,148.00,136.72,134.26,131.63,131.32,129.39$, 129.34, 122.22, 108.98, 108.67, 101.90, 44.62, 44.03, 40.48, 27.33, 15.37. IR (KBr, $\left.\mathrm{cm}^{-1}\right) 1670,1637,1489,1444,1296,1251,1035,926,813$.

5-Benzoyl-2-(4-chlorophenyl)-6-ethylthio-2,3-dihydrothiopyran-4-one (2c): $\mathrm{mp}$ : 127-129 ${ }^{\circ} \mathrm{C} ;{ }^{1} \mathrm{H}$ NMR $\left(500 \mathrm{MHz}, \mathrm{CDCl}_{3}\right) \delta=1.26(\mathrm{t}, J=7.5 \mathrm{~Hz}, 3 \mathrm{H}), 2.93-3.05(\mathrm{~m}$, $3 \mathrm{H}), 3.23(\mathrm{dd}, J=13.5,16.5 \mathrm{~Hz}, 1 \mathrm{H}), 4.80(\mathrm{dd}, J=3.0,13.5 \mathrm{~Hz}, 1 \mathrm{H}), 7.38-7.46(\mathrm{~m}$, $6 \mathrm{H}), 7.55-7.58(\mathrm{~m}, 1 \mathrm{H}), 7.85(\mathrm{dd}, J=1.0,8.0 \mathrm{~Hz}, 2 \mathrm{H}) . \mathrm{IR}\left(\mathrm{KBr}, \mathrm{cm}^{-1}\right) 1669,1636$, 1491, 1296, 1232, 1091, 856. HRMS-ESI (m/z): $[\mathrm{M}+\mathrm{K}]^{+}$calcd for $\mathrm{C}_{20} \mathrm{H}_{17} \mathrm{ClO}_{2} \mathrm{~S}_{2} \mathrm{~K}$, 426.9990; found 426.9987.

5-Benzoyl-2-(2-chlorophenyl)-6-ethylthio-2,3-dihydrothiopyran-4-one (2d): White semisolid; ${ }^{1} \mathrm{H}$ NMR (500 MHz, $\left.\mathrm{CDCl}_{3}\right) \delta=1.26(\mathrm{t}, J=7.5 \mathrm{~Hz}, 3 \mathrm{H}), 2.93-3.07(\mathrm{~m}$, $3 \mathrm{H}), 3.25(\mathrm{dd}, J=13.0,16.5 \mathrm{~Hz}, 1 \mathrm{H}), 5.03(\mathrm{dd}, J=3.0,13.0 \mathrm{~Hz}, 1 \mathrm{H}), 7.32-7.39(\mathrm{~m}$, 2H), 7.45-7.48 (m, 3H), 7.53-7.59 (m, 2H), $7.90(\mathrm{~d}, J=7.5 \mathrm{~Hz}, 2 \mathrm{H}) . \mathrm{IR}\left(\mathrm{KBr}, \mathrm{cm}^{-1}\right)$ $1660,1643,1534,1313,1290,1091,832$.

5-Benzoyl-6-ethylthio-2-(pyridin-3-yl)-2,3-dihydrothiopyran-4-one $\quad$ (2e): $\mathrm{mp}$ : $115-117{ }^{\circ} \mathrm{C} ;{ }^{1} \mathrm{H} \mathrm{NMR}\left(500 \mathrm{MHz}, \mathrm{CDCl}_{3}\right) \quad \delta=1.25(\mathrm{t}, J=7.5 \mathrm{~Hz}, 3 \mathrm{H}), 2.93-3.06(\mathrm{~m}$, $3 \mathrm{H}), 3.26(\mathrm{dd}, J=13.5,16.5 \mathrm{~Hz}, 1 \mathrm{H}), 4.84(\mathrm{dd}, J=2.0,13.5 \mathrm{~Hz}, 1 \mathrm{H}), 7.37-7.40(\mathrm{~m}$, 
1H), 7.43-7.46 (m, 2H), 7.55-7.58 (m, 1H), $7.79(\mathrm{~d}, J=8.0 \mathrm{~Hz}, 1 \mathrm{H}), 7.85(\mathrm{~d}, J=8.0$ $\mathrm{Hz}, 2 \mathrm{H}), 8.65(\mathrm{~d}, J=4.5 \mathrm{~Hz}, 1 \mathrm{H}), 8.73(\mathrm{~s}, 1 \mathrm{H}) .{ }^{13} \mathrm{C} \mathrm{NMR}\left(125 \mathrm{MHz}, \mathrm{CDCl}_{3}\right) \delta=$ $194.39,189.34,161.28,150.53,149.51,136.79,135.30,133.92,132.89,131.87$, 129.40, 128.97, 124.14, 44.56, 43.94, 27.93, 14.90. IR (KBr, $\left.\mathrm{cm}^{-1}\right)$ 1673, 1631, 1498 , 1297, 1232, 860, 814, 684. HRMS-ESI (m/z): $[\mathrm{M}+\mathrm{Na}]^{+}$calcd for $\mathrm{C}_{19} \mathrm{H}_{17} \mathrm{NO}_{2} \mathrm{~S}_{2} \mathrm{Na}$, 378.0593; found 378.0557.

(E)-5-benzoyl-6-ethylthio-2-styryl-2,3-dihydrothiopyran-4-one (2f): Yellowish semisolid; ${ }^{1} \mathrm{H}$ NMR $\left(500 \mathrm{MHz}, \mathrm{CDCl}_{3}\right) \delta=1.27(\mathrm{t}, J=7.5 \mathrm{~Hz}, 3 \mathrm{H}), 2.99-3.05(\mathrm{~m}$, 4H), 4.42-4.44 (m, 1H), $6.30(\mathrm{dd}, J=7.0,16.0 \mathrm{~Hz}, 1 \mathrm{H}), 6.75(\mathrm{~d}, J=16.0 \mathrm{~Hz}, 1 \mathrm{H})$, 7.31-7.39 (m, 5H), $7.43(\mathrm{~d}, J=7.5 \mathrm{~Hz}, 2 \mathrm{H}), 7.51-7.54(\mathrm{~m}, 1 \mathrm{H}), 7.87(\mathrm{~d}, J=7.5 \mathrm{~Hz}$, 2H). ${ }^{13} \mathrm{C}$ NMR $\left(125 \mathrm{MHz}, \mathrm{CDCl}_{3}\right) \delta=194.14,189.21,160.45,136.46,135.20$, $134.36,133.24,131.51,128.93,128.55,128.43,128.38,126.48,124.16,44.66,42.92$, $27.40,14.45$.

5-Benzoyl-6-methylthio-2-(4-chlorophenyl)-2,3-dihydrothiopyran-4-one (2g): mp: 101-103 ${ }^{\circ} \mathrm{C} ;{ }^{1} \mathrm{H}$ NMR $\left(500 \mathrm{MHz}, \mathrm{CDCl}_{3}\right) \delta=2.46(\mathrm{~s}, 3 \mathrm{H}), 2.30(\mathrm{dd}, J=3.0,16.5 \mathrm{~Hz}$, $1 \mathrm{H}), 3.22(\mathrm{dd}, J=9.0,16.5 \mathrm{~Hz}, 1 \mathrm{H}), 4.80(\mathrm{dd}, J=3.0,9.0 \mathrm{~Hz}, 1 \mathrm{H}), 7.37-7.47(\mathrm{~m}, 6 \mathrm{H})$, 7.55-7.59 (m, 1H), $7.85(\mathrm{~d}, J=7.5 \mathrm{~Hz}, 2 \mathrm{H})$.

\section{2-(4-Chlorophenyl)-5-(4-ethoxybenzoyl)-6-ethylthio-2,3-dihydrothiopyran-4-one}

(2h): mp: $158-159{ }^{\circ} \mathrm{C} ;{ }^{1} \mathrm{H}$ NMR $\left(500 \mathrm{MHz}, \mathrm{CDCl}_{3}\right) \delta=1.25(\mathrm{t}, J=7.5 \mathrm{~Hz}, 3 \mathrm{H}), 1.44$ $(\mathrm{t}, J=7.5 \mathrm{~Hz}, 3 \mathrm{H}), 2.92-3.04(\mathrm{~m}, 3 \mathrm{H}), 3.22(\mathrm{dd}, J=13.5,16.5 \mathrm{~Hz}, 1 \mathrm{H}), 4.10(\mathrm{q}, J=$ $7.5 \mathrm{~Hz}, 2 \mathrm{H}), 4.77(\mathrm{dd}, J=2.5,13.5 \mathrm{~Hz}, 1 \mathrm{H}), 6.91(\mathrm{~d}, J=9.0 \mathrm{~Hz}, 2 \mathrm{H}), 7.37-7.42(\mathrm{~m}$, 
4H), $7.82(\mathrm{~d}, J=9.0 \mathrm{~Hz}, 2 \mathrm{H}) .{ }^{13} \mathrm{C} \mathrm{NMR}\left(125 \mathrm{MHz}, \mathrm{CDCl}_{3}\right) \delta=192.44,189.17$, $163.25,160.00,135.09,134.54,131.65,131.40,129.23,129.11,128.83,127.87$, $114.12,63.55,45.90,43.85,27.34,14.46,14.41$.

\section{5-(4-Chlorobenzoyl)-2-(4-chlorophenyl)-6-ethylthio-2,3-dihydrothiopyran-4-one}

(2i): mp: $152-155{ }^{\circ} \mathrm{C} ;{ }^{1} \mathrm{H}$ NMR $\left(500 \mathrm{MHz}, \mathrm{CDCl}_{3}\right) \delta=1.27(\mathrm{t}, J=7.5 \mathrm{~Hz}, 3 \mathrm{H})$, 2.97-3.04 (s, 3H), $3.23(\mathrm{dd}, J=13.5,16.5 \mathrm{~Hz}, 1 \mathrm{H}), 4.79(\mathrm{dd}, J=3.0,13.5 \mathrm{~Hz}, 1 \mathrm{H})$, 7.38-7.43 (m, 6H), $7.78(\mathrm{~d}, J=8.5 \mathrm{~Hz}, 2 \mathrm{H}) .{ }^{13} \mathrm{C} \mathrm{NMR}\left(125 \mathrm{MHz}, \mathrm{CDCl}_{3}\right) \delta=192.07$, $188.41,161.22,138.97,134.15,134.01,129.83,129.51,128.46,128.03,45.29,43.03$, 26.62, 13.59. IR (KBr, $\left.\mathrm{cm}^{-1}\right) 1683,1670,1584,1491,1311,1091,1013,856$.

6-ethylthio-2-(p-tolyl)-2,3-dihydrothiopyran-4-one (2j): mp: $58-60{ }^{\circ} \mathrm{C} ;{ }^{1} \mathrm{H}$ NMR $\left(500 \mathrm{MHz}, \mathrm{CDCl}_{3}\right) \delta=1.36(\mathrm{t}, J=7.5 \mathrm{~Hz}, 3 \mathrm{H}), 2.36(\mathrm{~s}, 3 \mathrm{H}), 2.92(\mathrm{dd}, \mathrm{J}=3.0,16.5$ $\mathrm{Hz}, 1 \mathrm{H}), 2.94-3.06(\mathrm{~m}, 2 \mathrm{H}), 3.10(\mathrm{dd}, J=14.5,16.5 \mathrm{~Hz}, 1 \mathrm{H}), 4.66(\mathrm{dd}, J=3.0,14.5$ $\mathrm{Hz}, 1 \mathrm{H}), 6.26(\mathrm{~s}, 1 \mathrm{H}), 7.20(\mathrm{~d}, J=8.0 \mathrm{~Hz}, 2 \mathrm{H}), 7.27(\mathrm{~d}, J=8.0 \mathrm{~Hz}, 2 \mathrm{H}) . \mathrm{IR}(\mathrm{KBr}$, $\left.\mathrm{cm}^{-1}\right) 2933,1652,1523,1456,1261$.

2-(Benzo[d][1,3]dioxol-5-yl)-6-ethylthio-2,3-dihydrothiopyran-4-one

(2k):

Semisolid; ${ }^{1} \mathrm{H}$ NMR $\left(500 \mathrm{MHz}, \mathrm{CDCl}_{3}\right) \delta=1.36(\mathrm{t}, J=7.5 \mathrm{~Hz}, 3 \mathrm{H}), 2.90(\mathrm{dd}, J=3.0$, $16.5 \mathrm{~Hz}, 1 \mathrm{H}), 2.93-3.06(\mathrm{~m}, 3 \mathrm{H}), 4.60(\mathrm{dd}, J=3.0,14.0 \mathrm{~Hz}, 1 \mathrm{H}), 5.98(\mathrm{~s}, 2 \mathrm{H}), 6.25(\mathrm{~s}$, 1H), $6.79(\mathrm{~d}, J=8.0 \mathrm{~Hz}, 1 \mathrm{H}), 6.82(\mathrm{~d}, J=8.0 \mathrm{~Hz}, 1 \mathrm{H}), 6.86(\mathrm{~s}, 1 \mathrm{H}) .{ }^{13} \mathrm{C}$ NMR $(125$ $\left.\mathrm{MHz}, \mathrm{CDCl}_{3}\right) \delta=190.93,162.22,147.08,146.88,129.86,120.28,116.82,107.59$, 106.81, 100.42, 46.67, 44.05, 26.13. 12.78. IR (KBr, $\left.\mathrm{cm}^{-1}\right)$ 1647, 1544, 1481, 1399, 1326, 1207, 1090, 861. 
2-(4-Chlorophenyl)-6-ethylthio-2,3-dihydrothiopyran-4-one (2l): $\mathrm{mp}: 81-84{ }^{\circ} \mathrm{C}$;

${ }^{1} \mathrm{H}$ NMR $\left(500 \mathrm{MHz}, \mathrm{CDCl}_{3}\right) \delta=1.35(\mathrm{t}, J=7.5 \mathrm{~Hz}, 3 \mathrm{H}), 2.92(\mathrm{dd}, J=2.5,16.0 \mathrm{~Hz}$, 1H), 2.93-3.03 (m, 2H), $3.06(\mathrm{dd}, J=14.0,16.0 \mathrm{~Hz}, 1 \mathrm{H}), 4.65(\mathrm{dd}, J=2.5,14.0 \mathrm{~Hz}$, 1H), $6.25(\mathrm{~s}, 1 \mathrm{H}), 7.31(\mathrm{~d}, J=8.5 \mathrm{~Hz}, 2 \mathrm{H}), 7.36(\mathrm{~d}, J=8.5 \mathrm{~Hz}, 2 \mathrm{H}) . \mathrm{IR}\left(\mathrm{KBr}, \mathrm{cm}^{-1}\right)$ 1642, 1520, 1263, 1088, 828. HRMS-ESI (m/z): $[2 \mathrm{M}+\mathrm{Na}]^{+}$calcd for $\left[2 \mathrm{C}_{13} \mathrm{H}_{13} \mathrm{ClOS}_{2}\right.$ + Na] 591.0085; found 591.0117.

\section{5-Benzoyl-2-(4-chlorophenyl)-6-ethylamino-2,3-dihydrothiopyran-4-one}

(3cb):

mp: $150-151{ }^{\circ} \mathrm{C} ;{ }^{1} \mathrm{H}$ NMR $\left(500 \mathrm{MHz}, \mathrm{CDCl}_{3}\right) \delta=1.36(\mathrm{t}, J=7.0 \mathrm{~Hz}, 3 \mathrm{H}), 2.89(\mathrm{dd}, J$ $=10.5,14.5 \mathrm{~Hz}, 1 \mathrm{H}), 3.44-3.49(\mathrm{~m}, 2 \mathrm{H}), 3.56(\mathrm{dd}, J=3.5,14.5 \mathrm{~Hz}, 1 \mathrm{H}), 4.08(\mathrm{dd}, J=$ 3.5, $10.5 \mathrm{~Hz}, 1 \mathrm{H}), 7.19(\mathrm{~d}, J=8.5 \mathrm{~Hz}, 2 \mathrm{H}), 7.29(\mathrm{~d}, J=8.5 \mathrm{~Hz}, 2 \mathrm{H}), 7.39-7.42(\mathrm{~m}$, 2H), 7.46-7.49 (m, 1H), 7.56 (d, $J=7.5 \mathrm{~Hz}, 2 \mathrm{H}), 10.96$ (br s, 1H). ${ }^{13} \mathrm{C}$ NMR $(125$ $\left.\mathrm{MHz}, \mathrm{CDCl}_{3}\right) \delta=191.56,182.10,138.21,135.24,131.95,130.12,129.90,129.53$, 128.29, 127.30, 127.19, 126.48, 103.26, 54.87, 40.07, 37.13, 13.30. IR $\left(\mathrm{KBr}, \mathrm{cm}^{-1}\right)$ 3215, 1669, 1590, 1490, 1414, 1357, 1297, 1092, 1014, 808, 768. ES-MS: (m/z): $372.3[\mathrm{M}+1]^{+}$. 


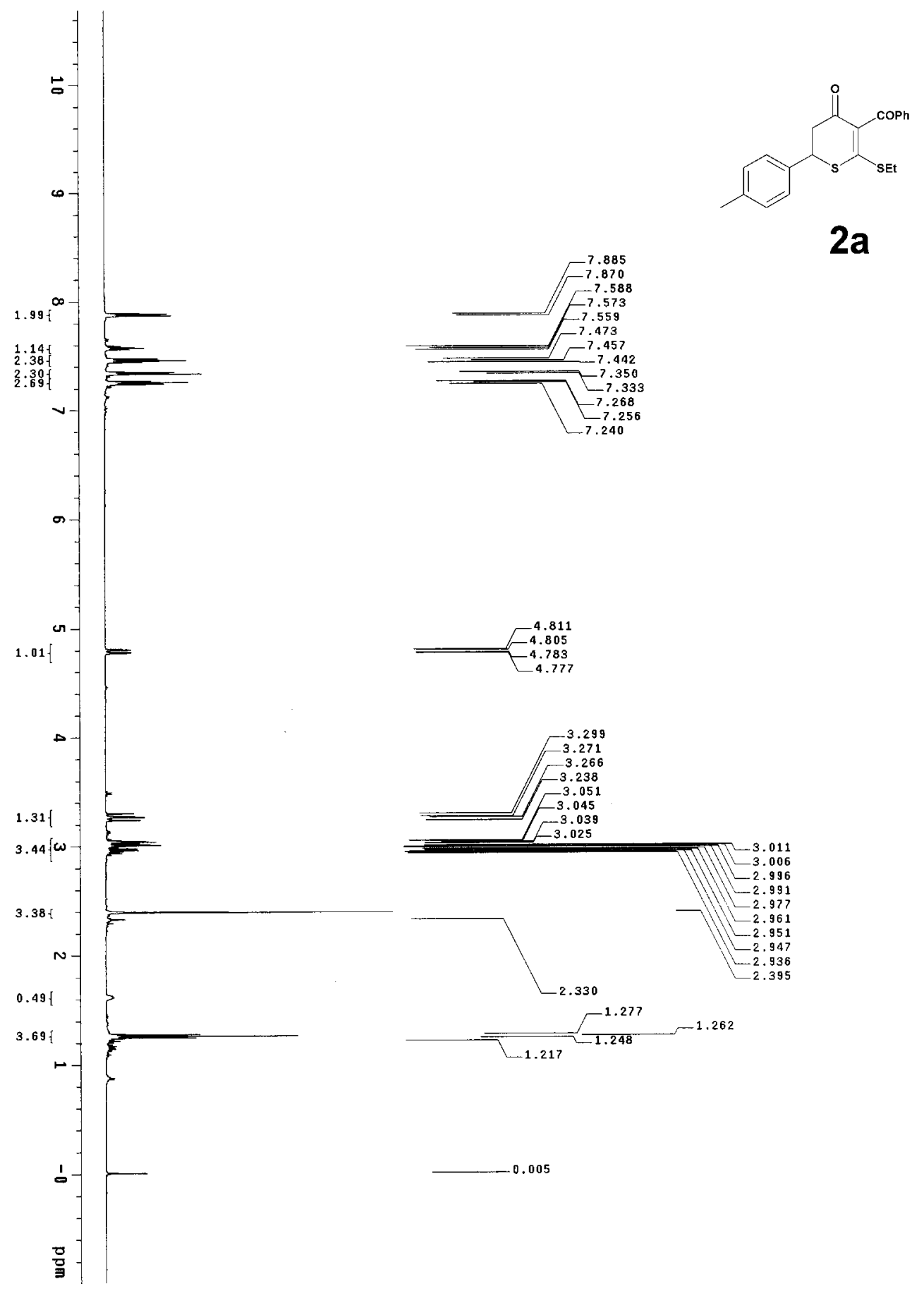




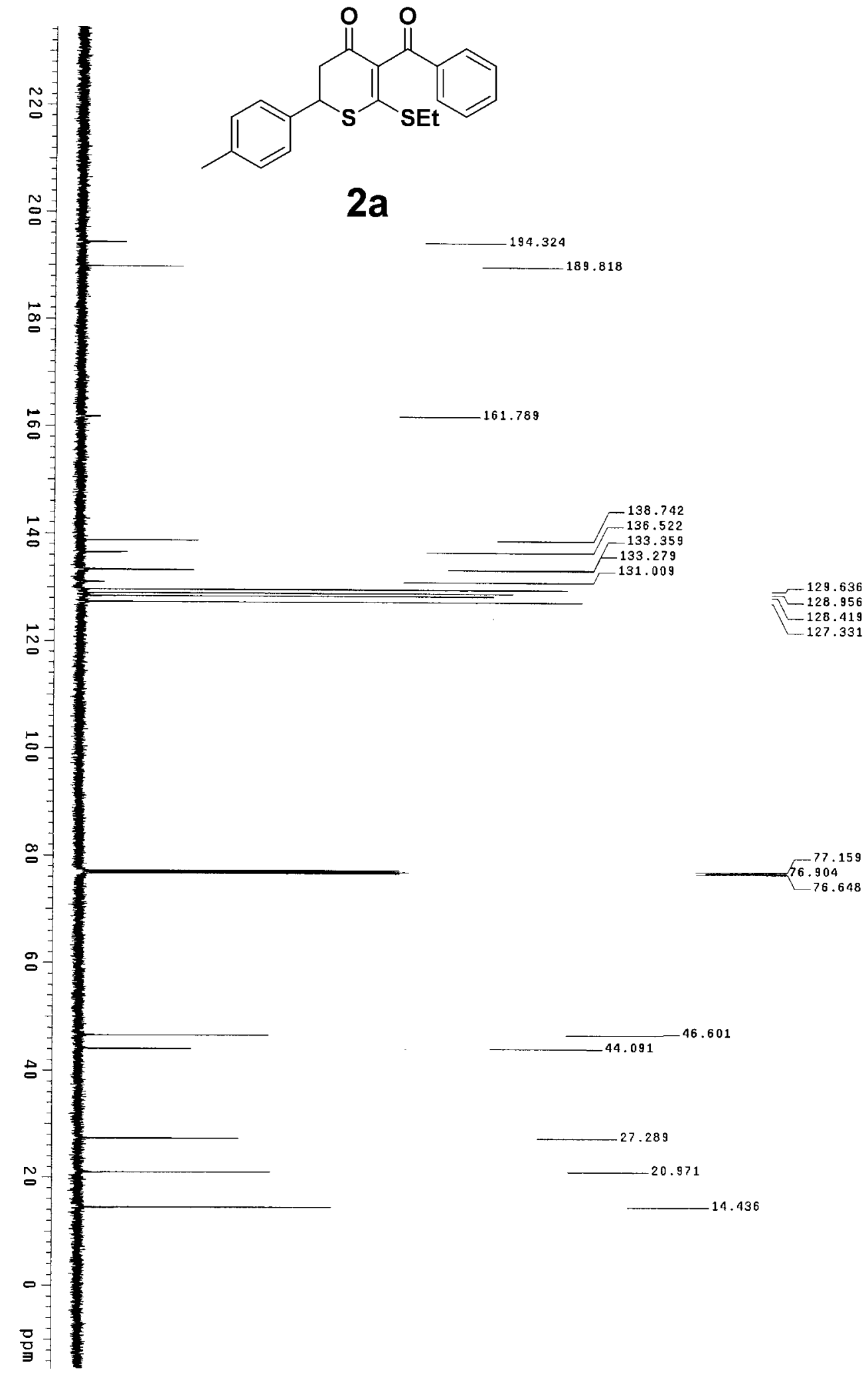




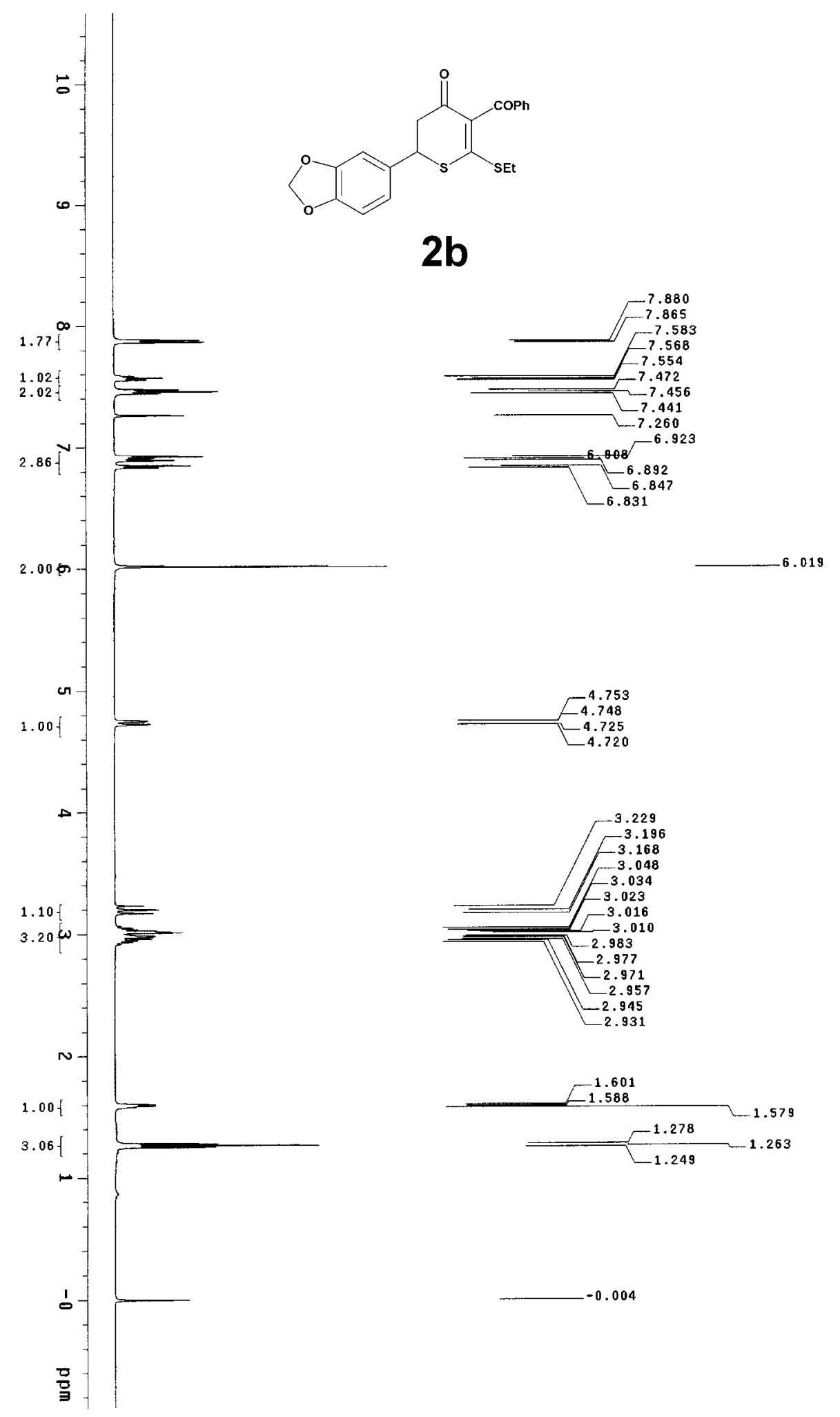




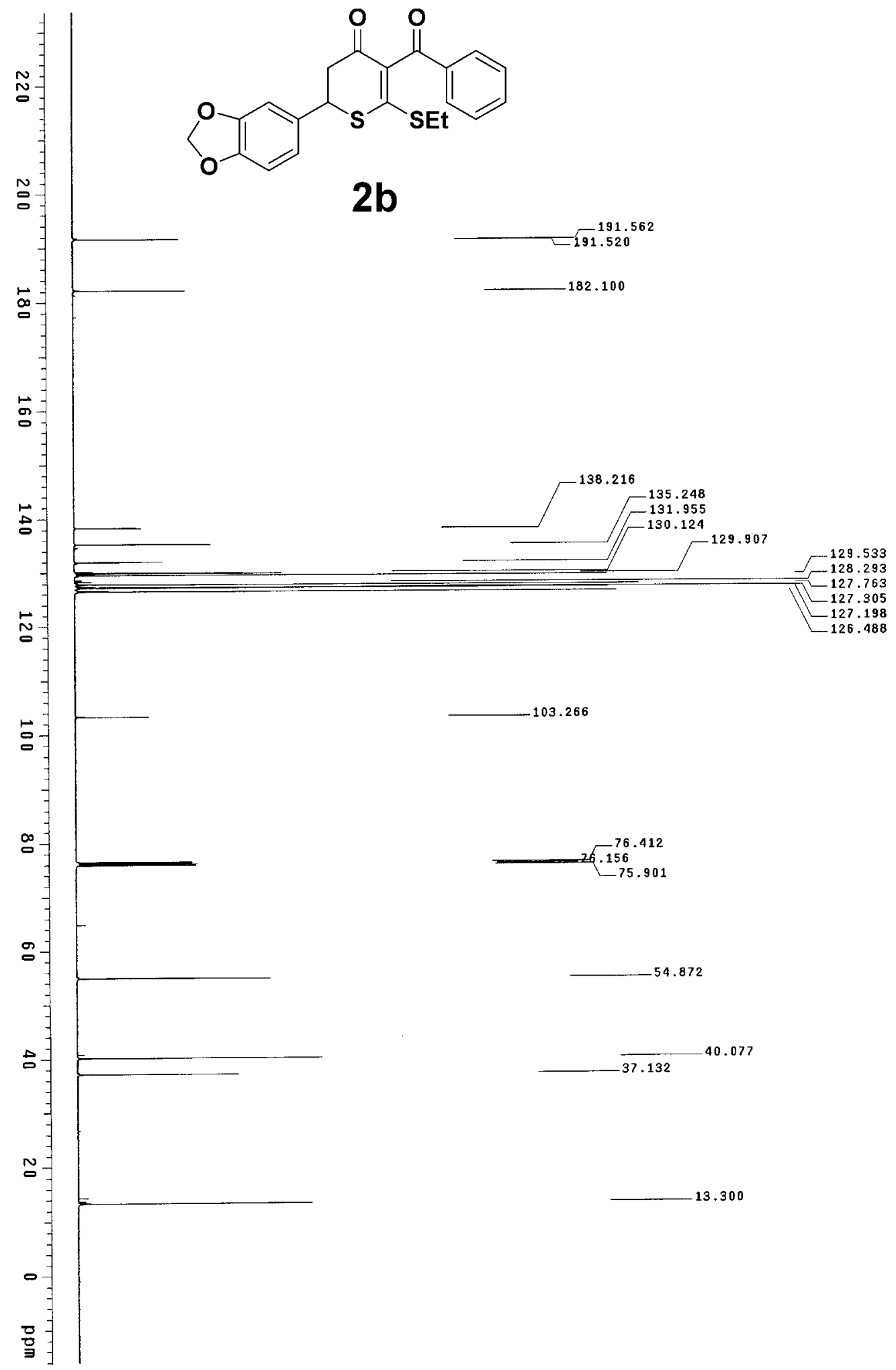




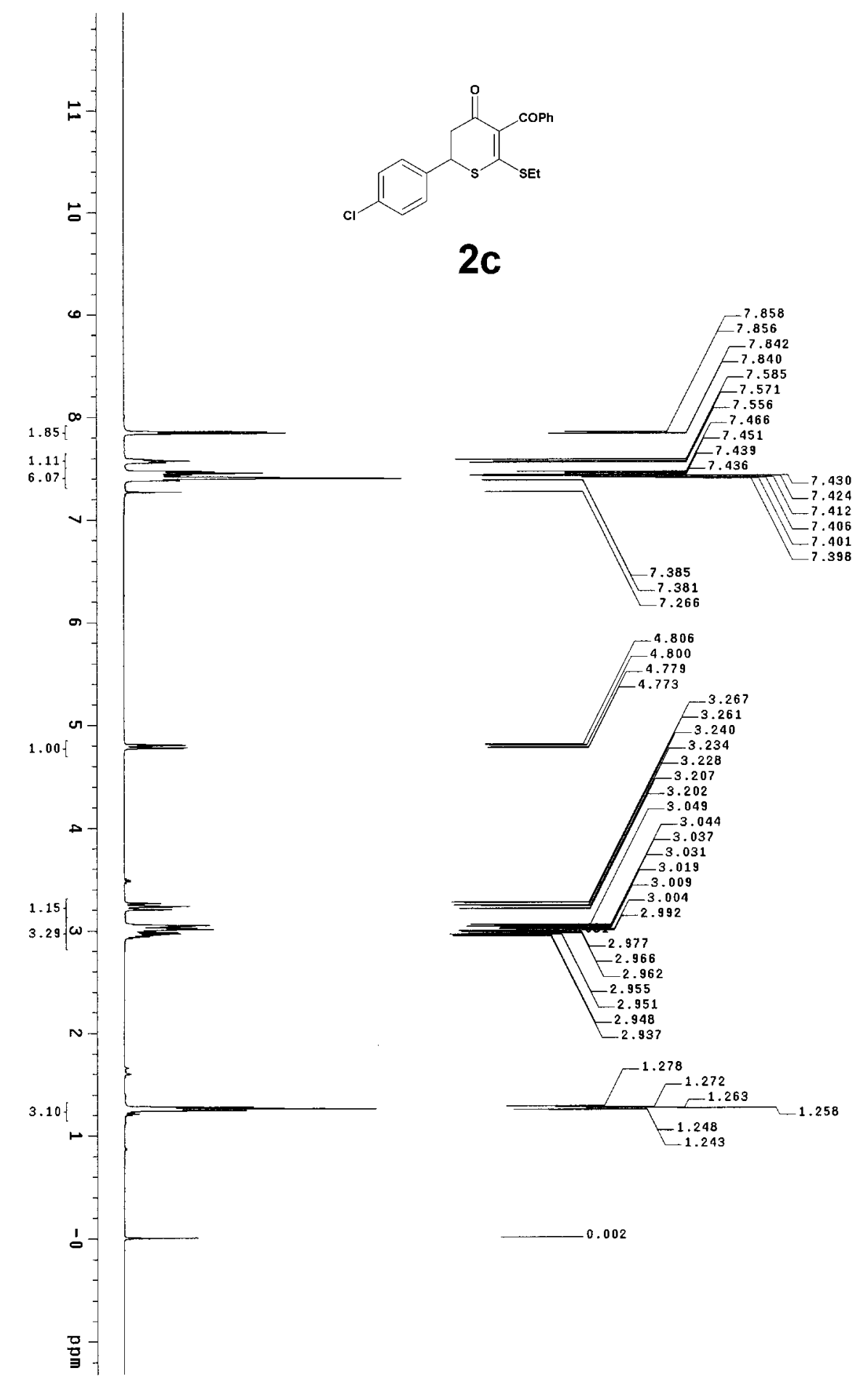




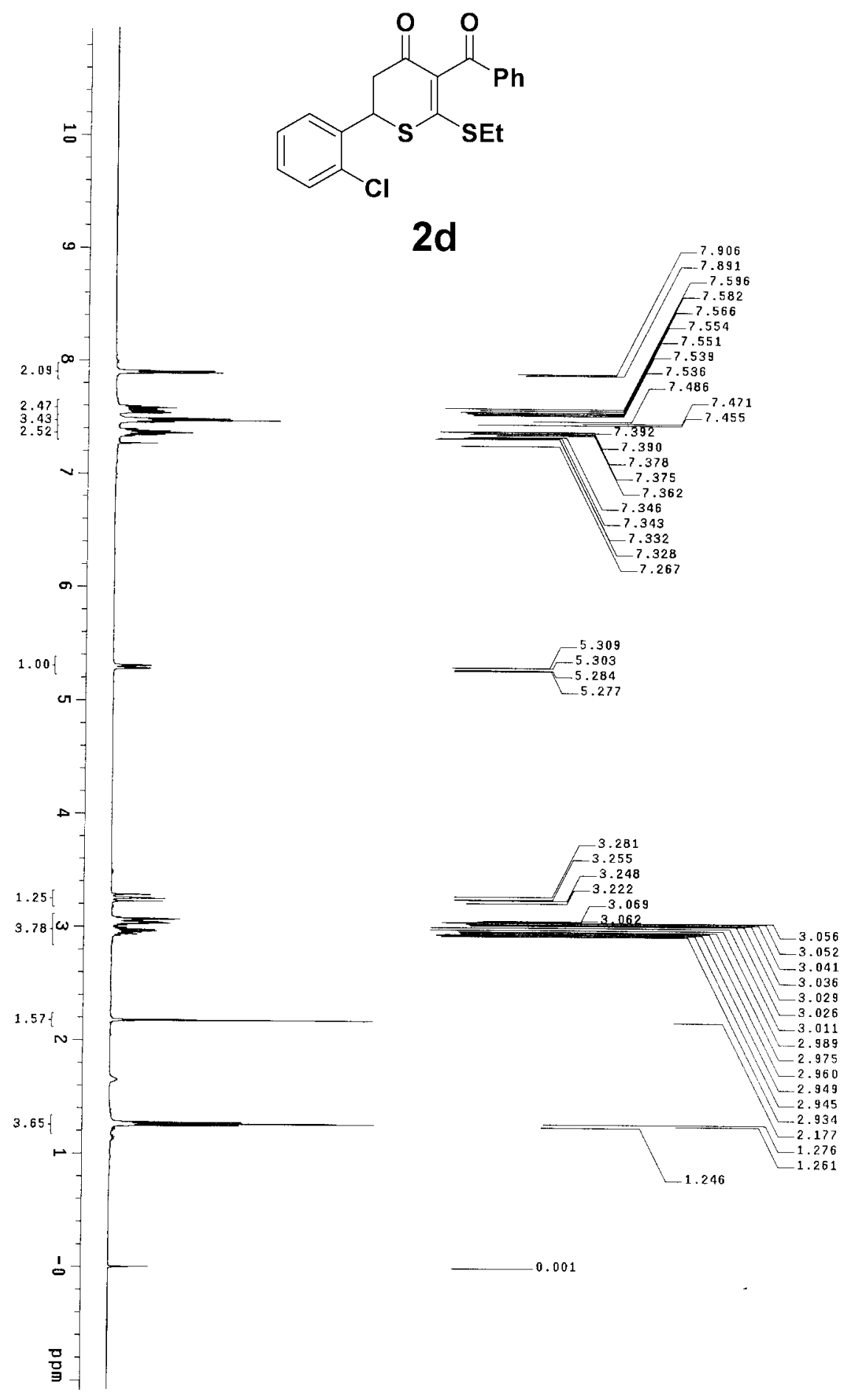



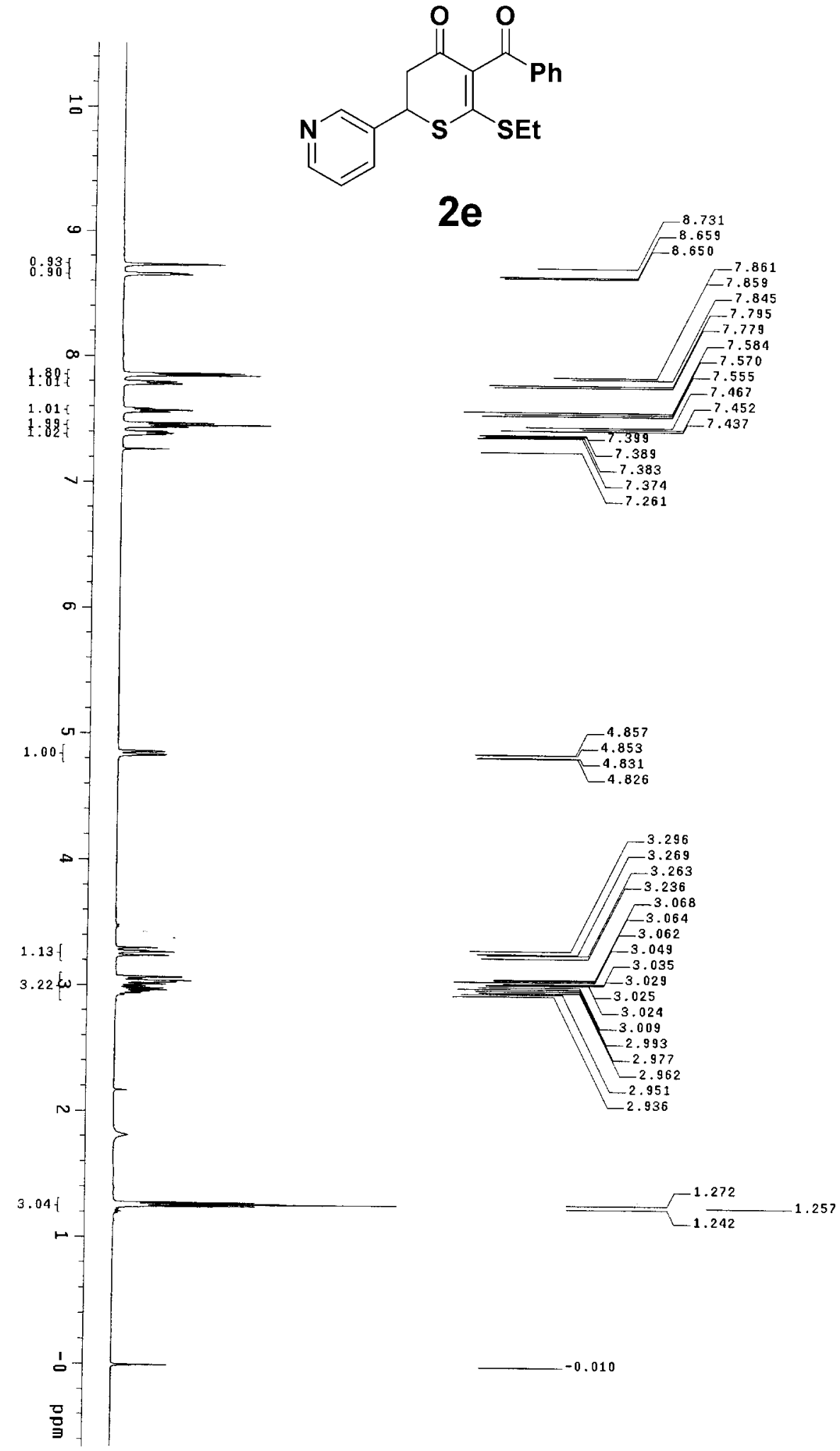


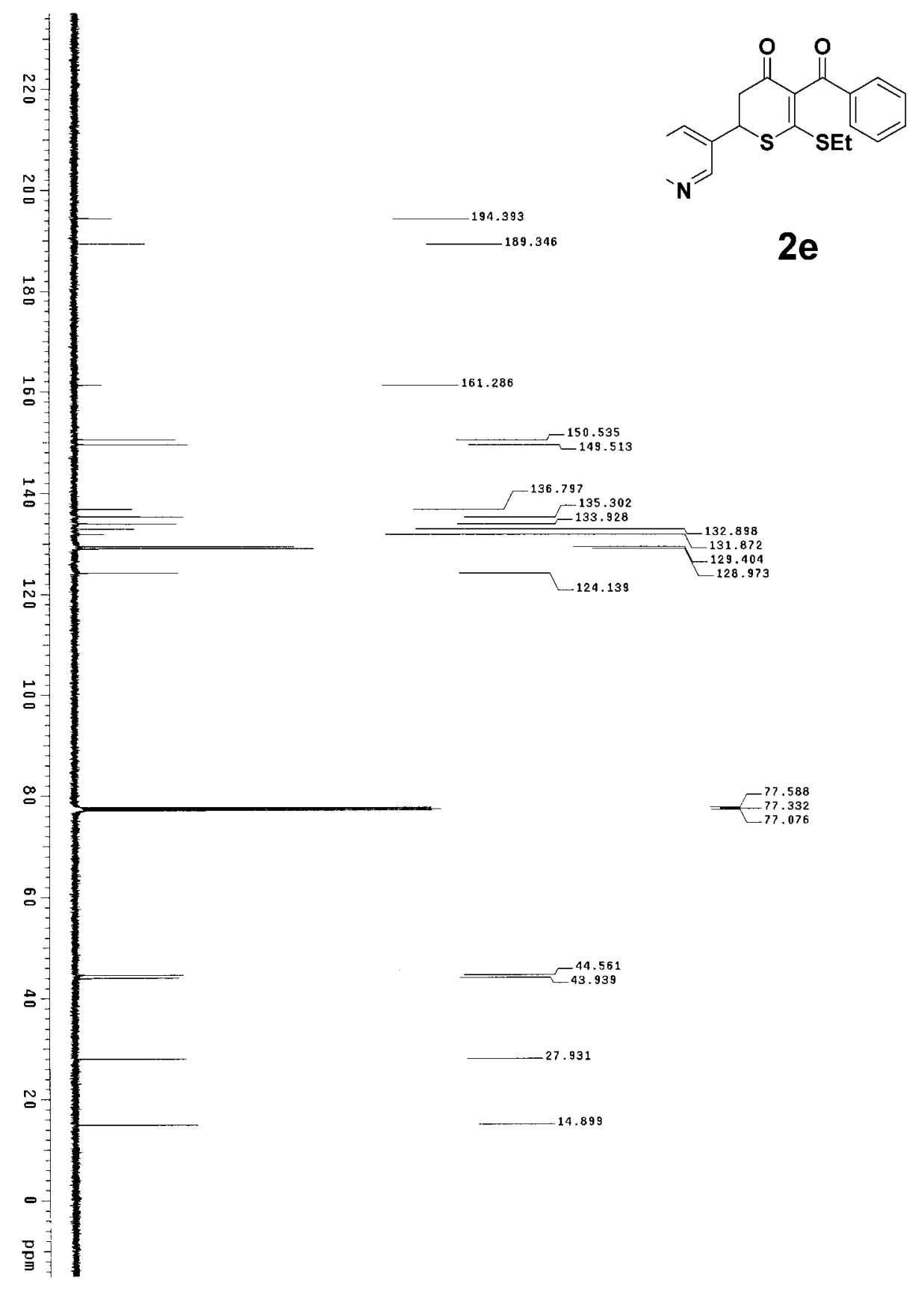




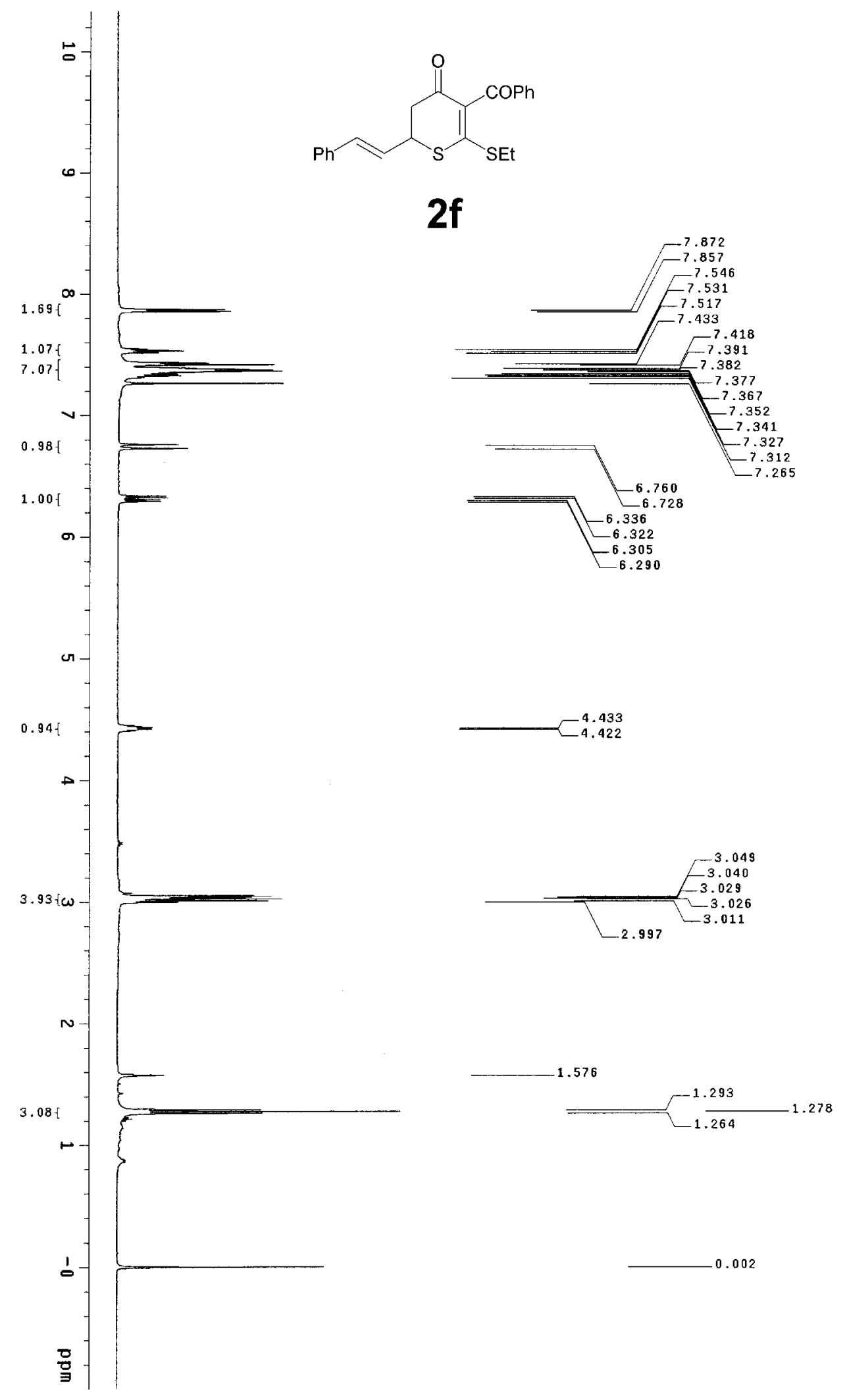




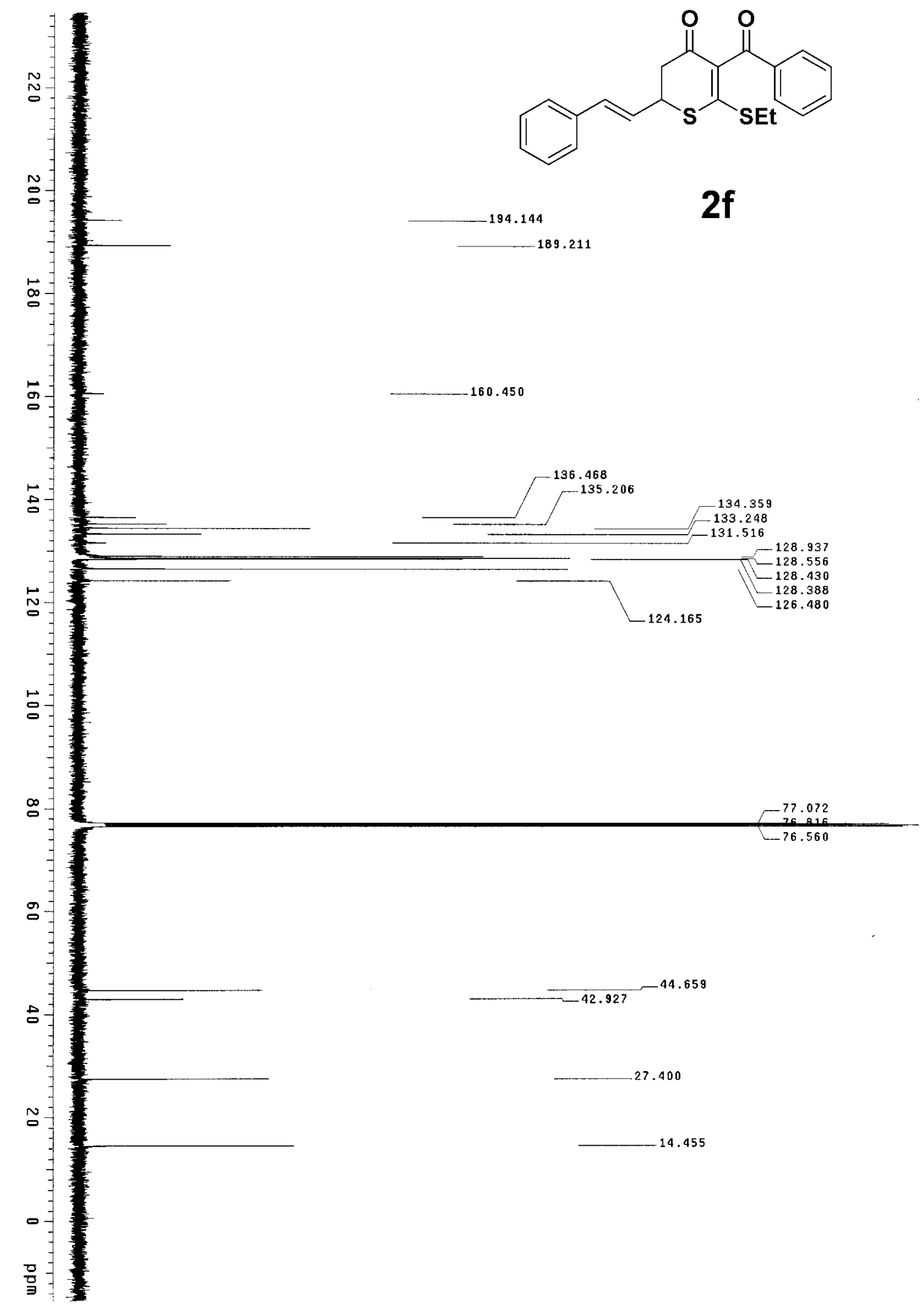




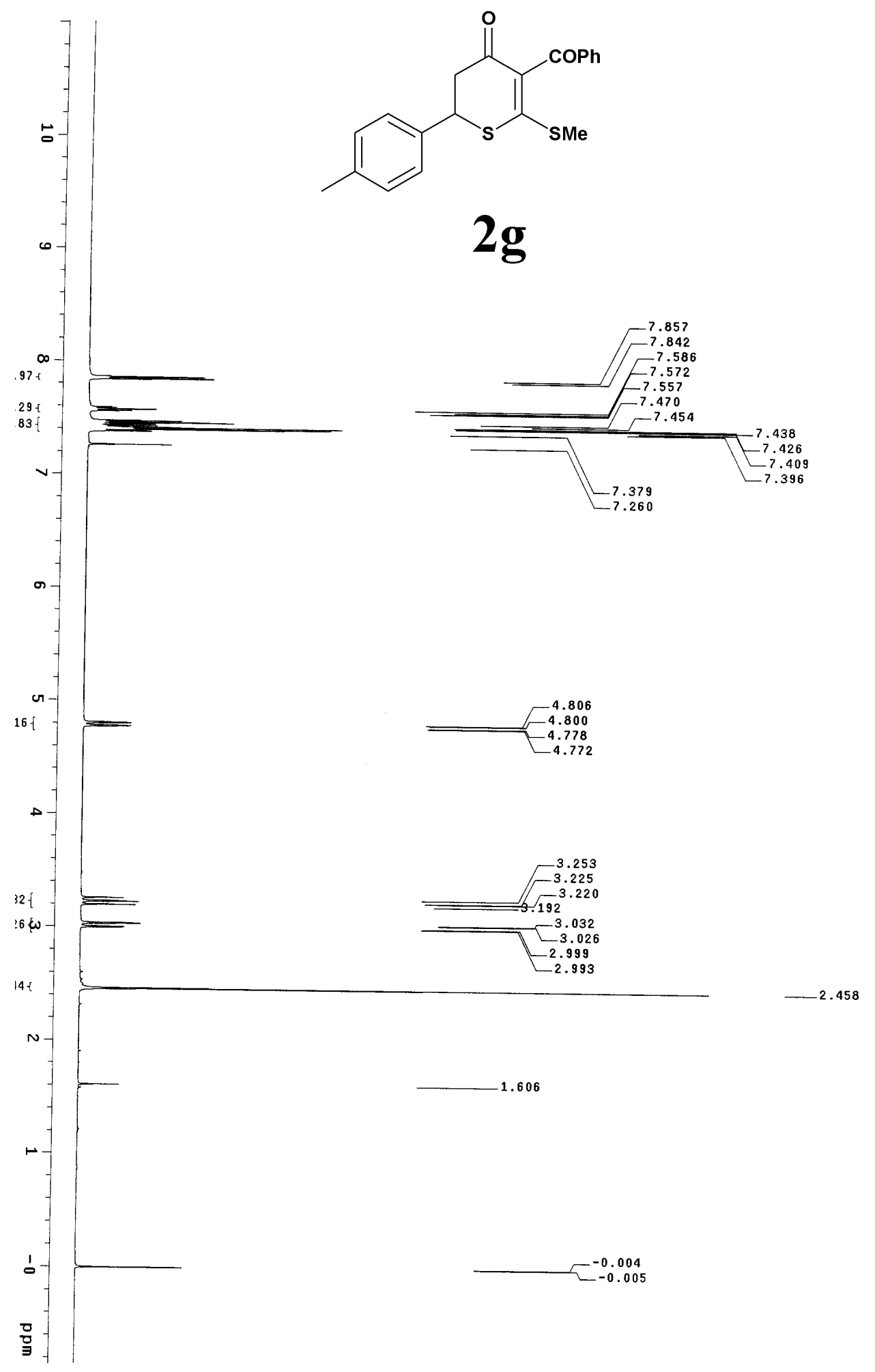




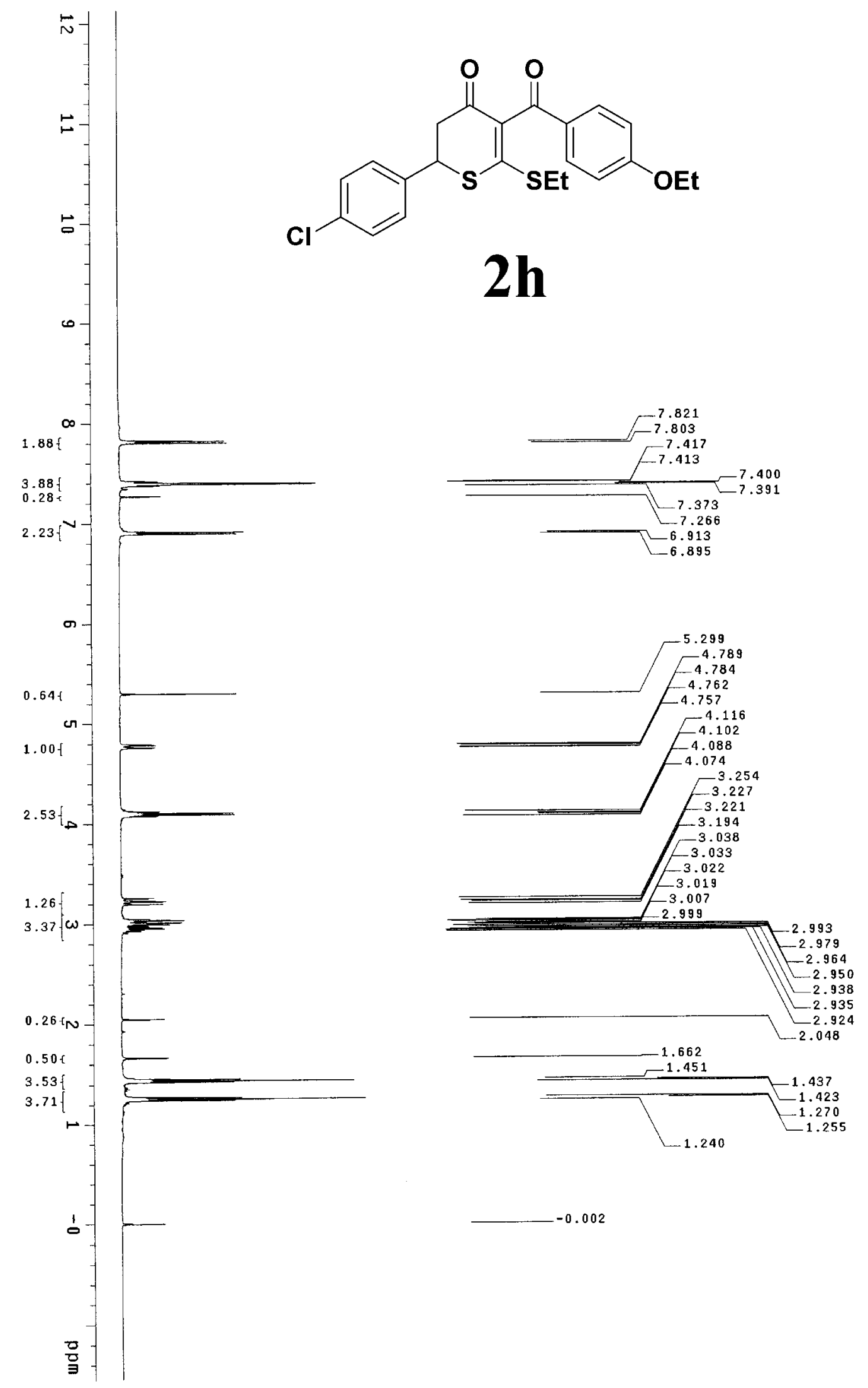




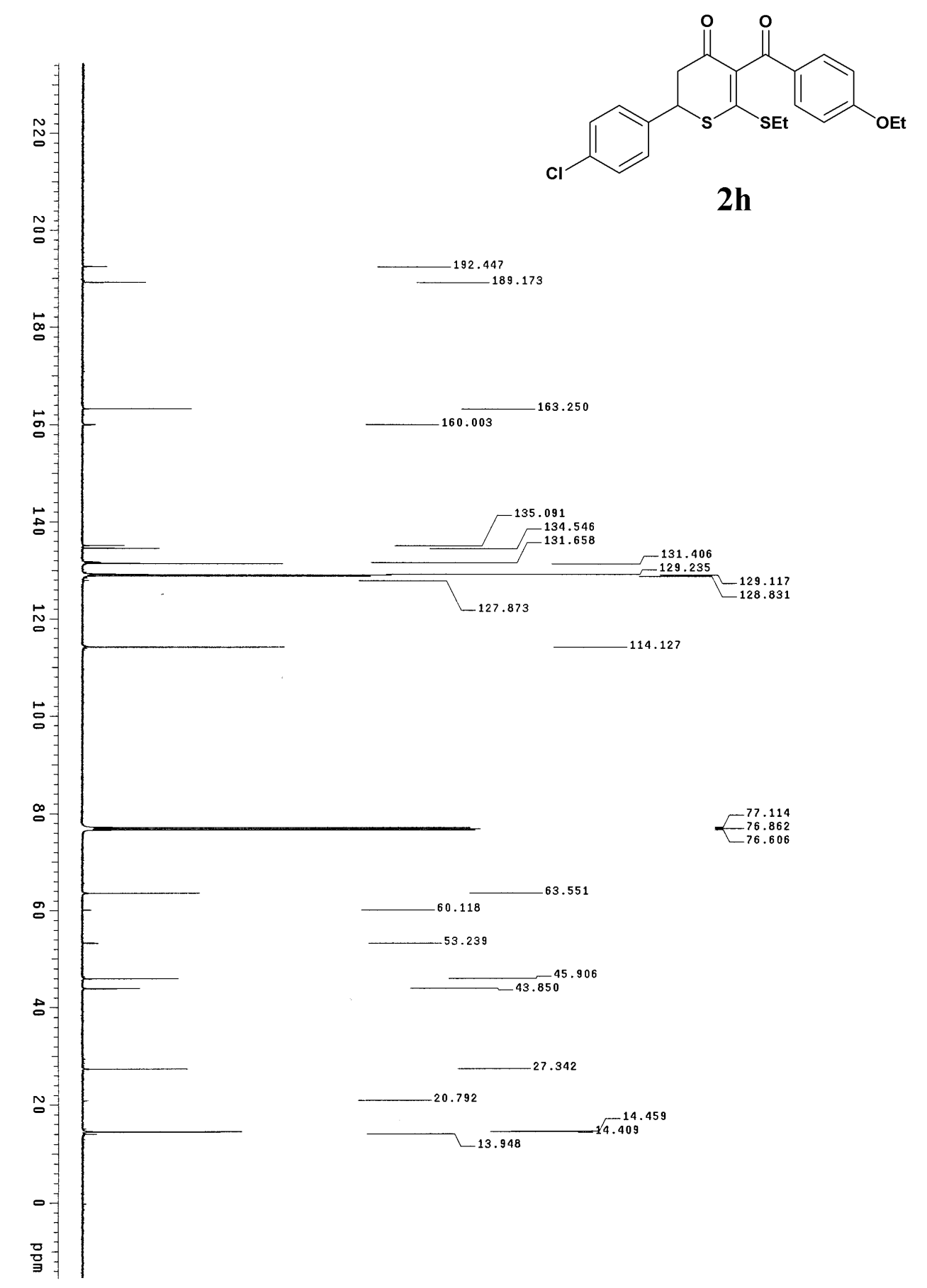




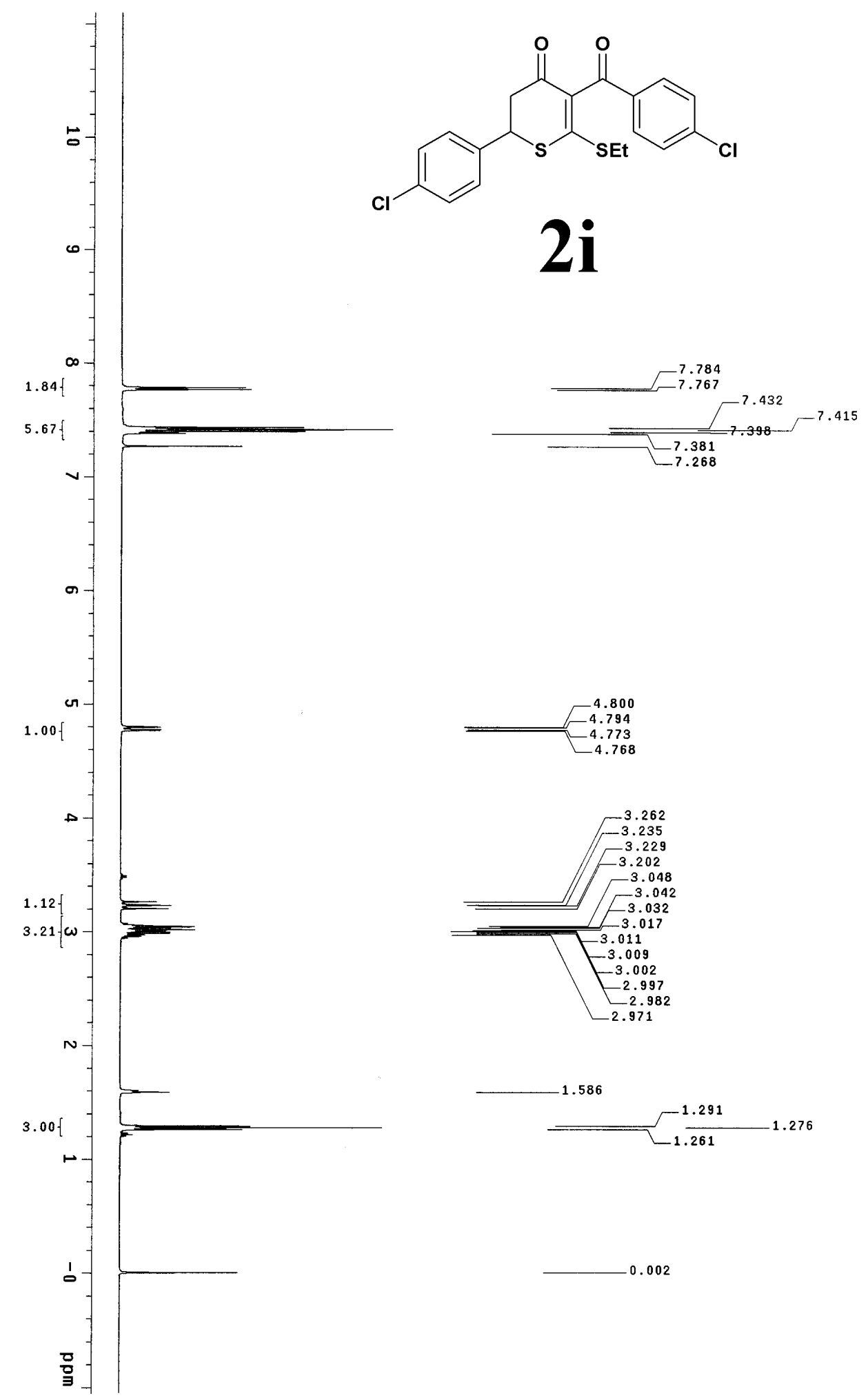




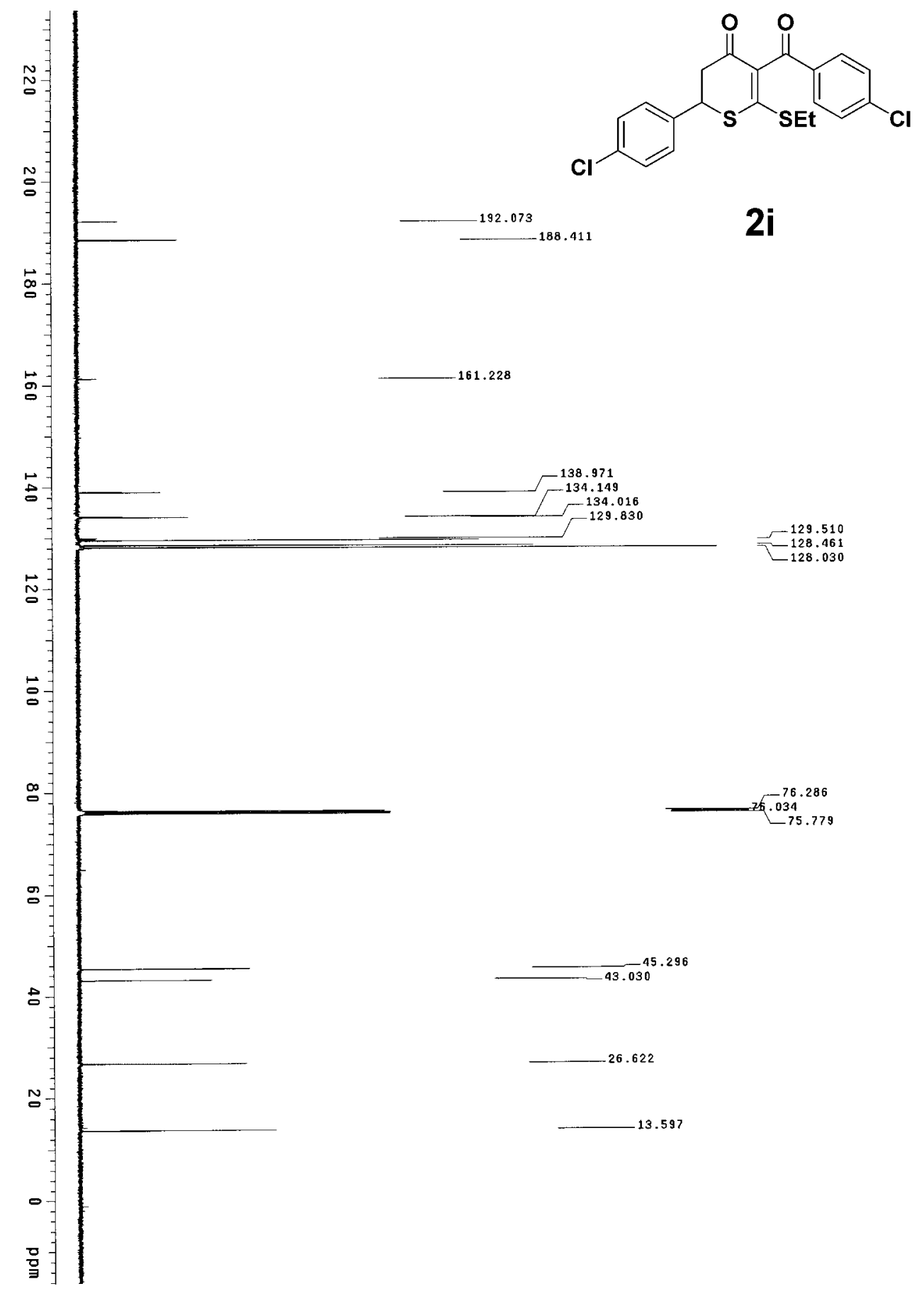




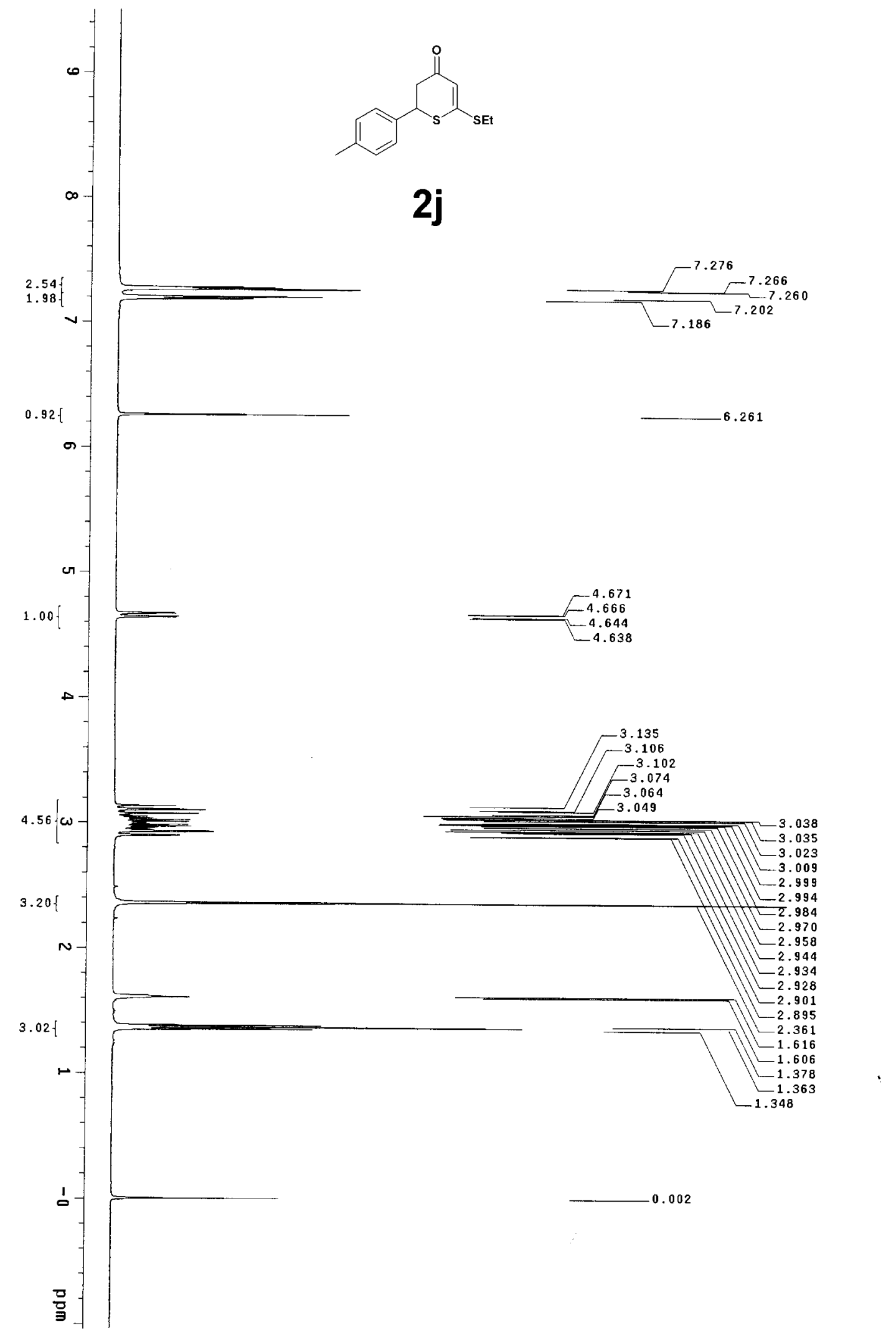




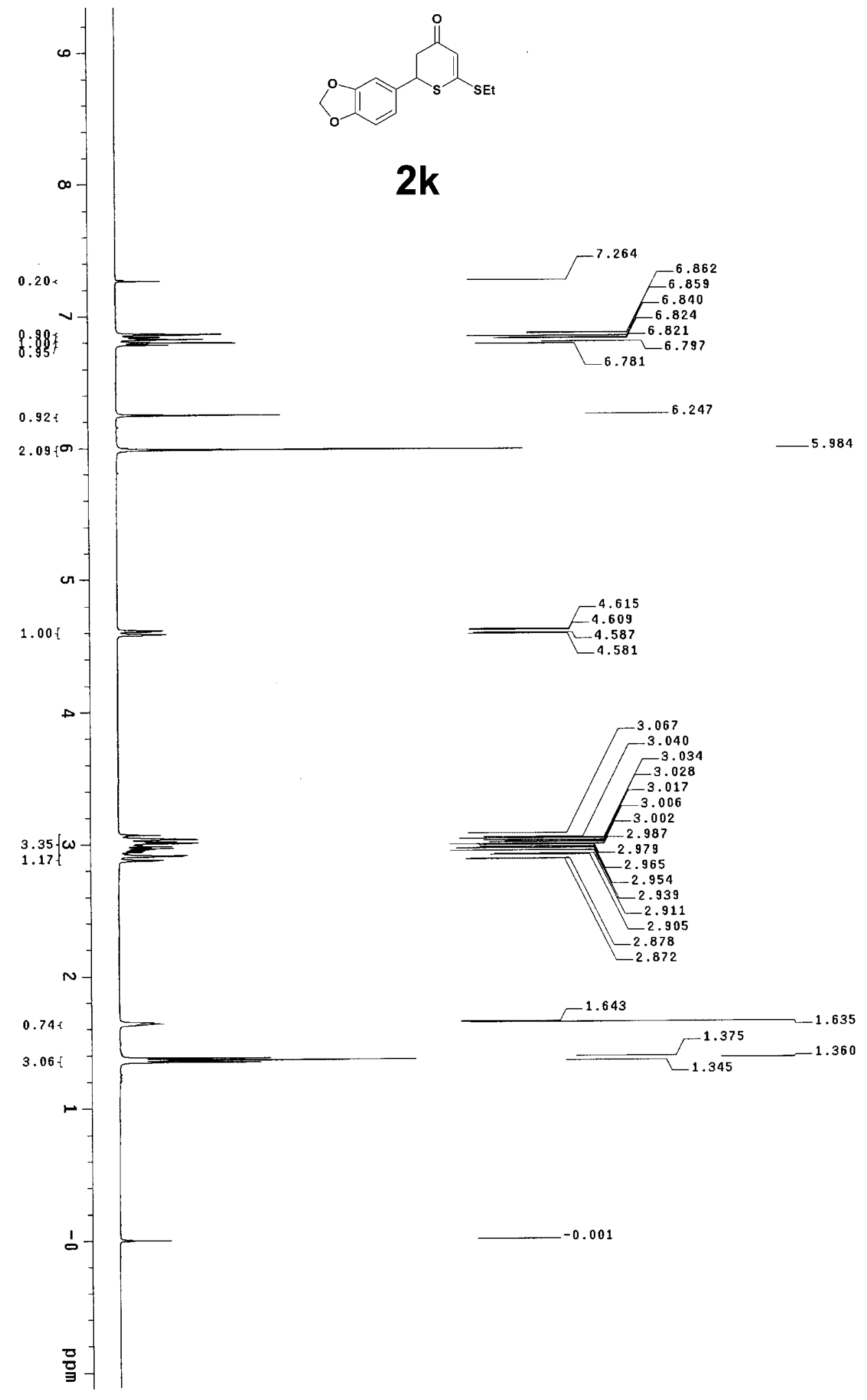




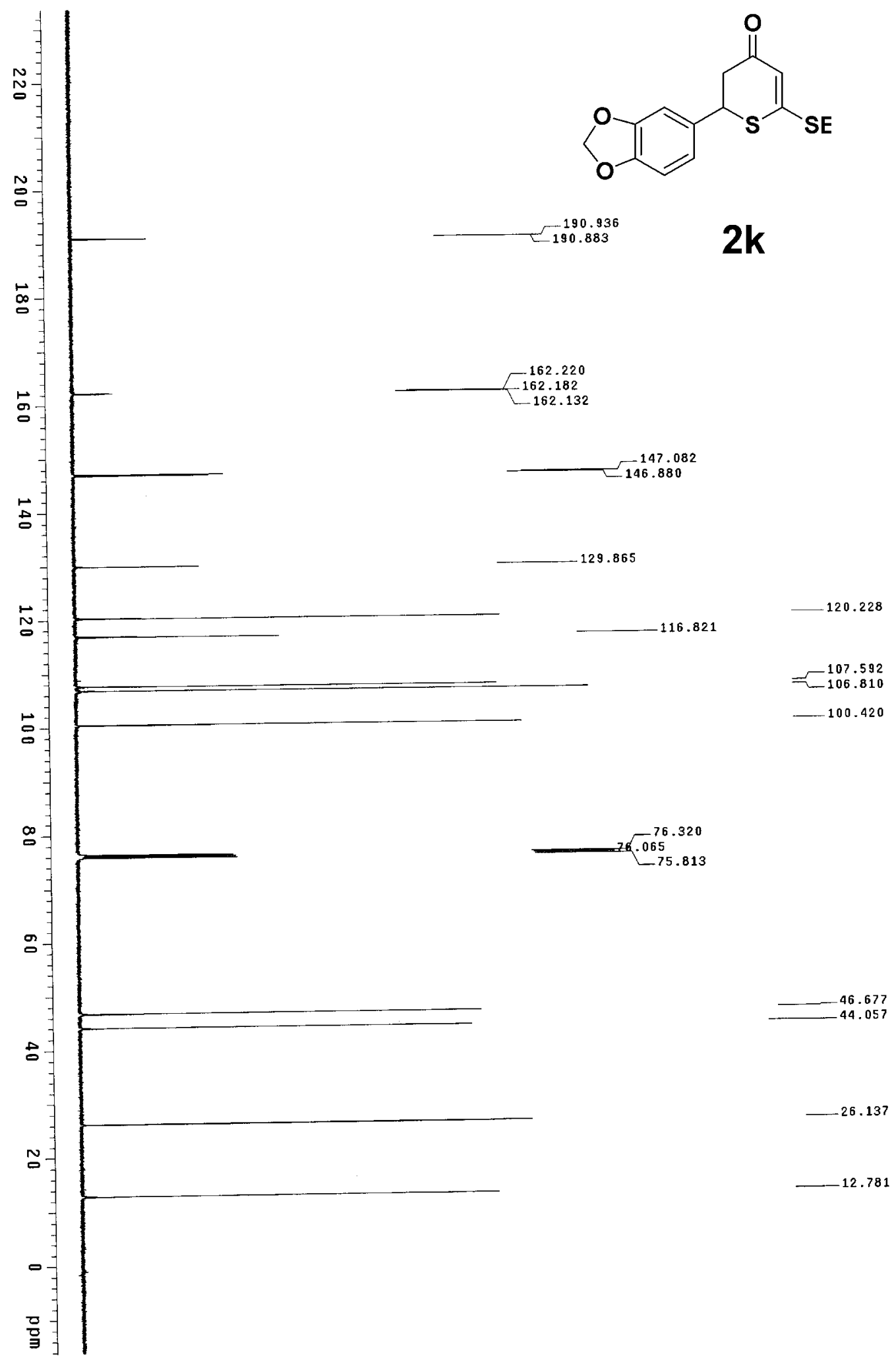




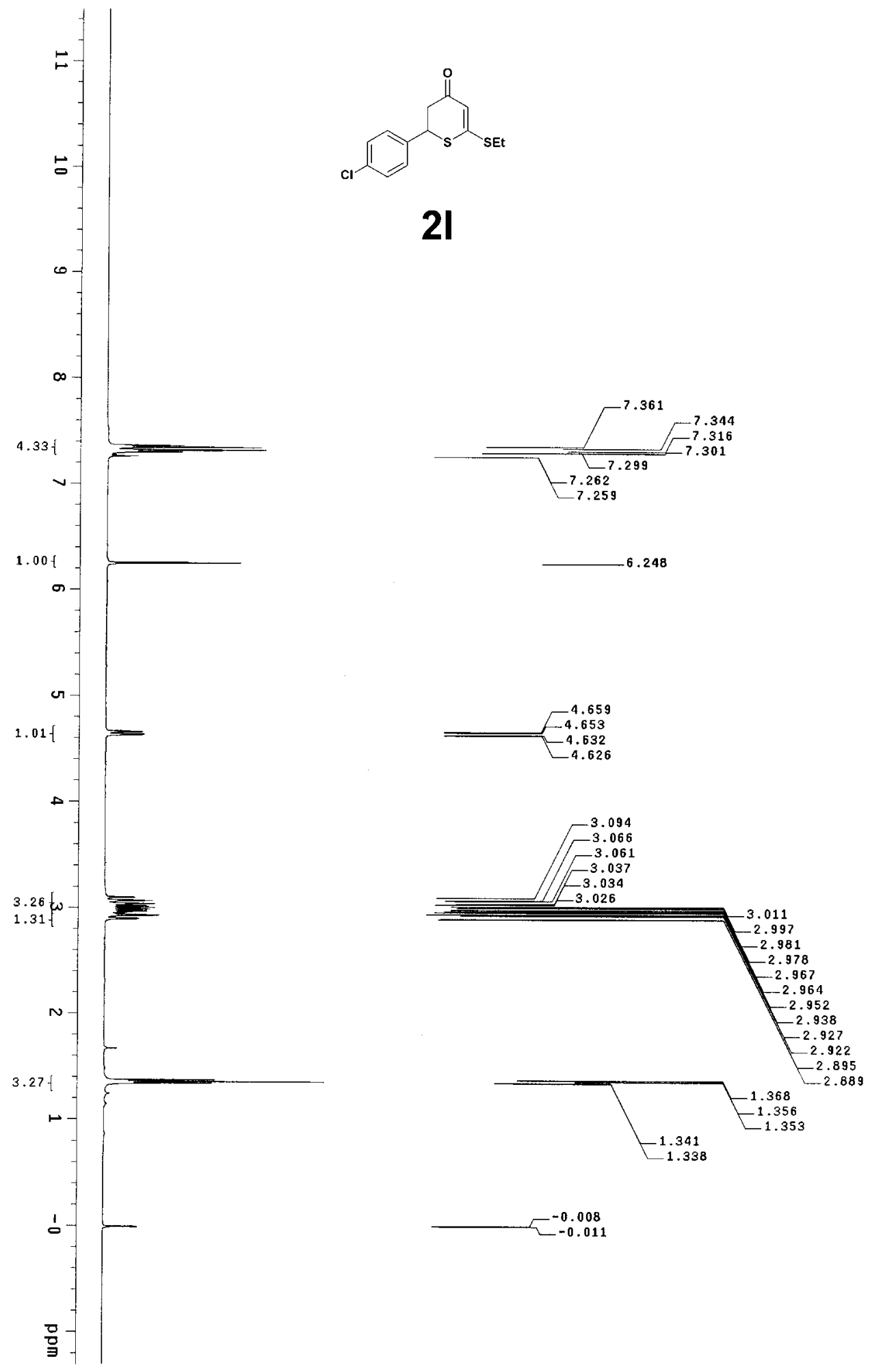




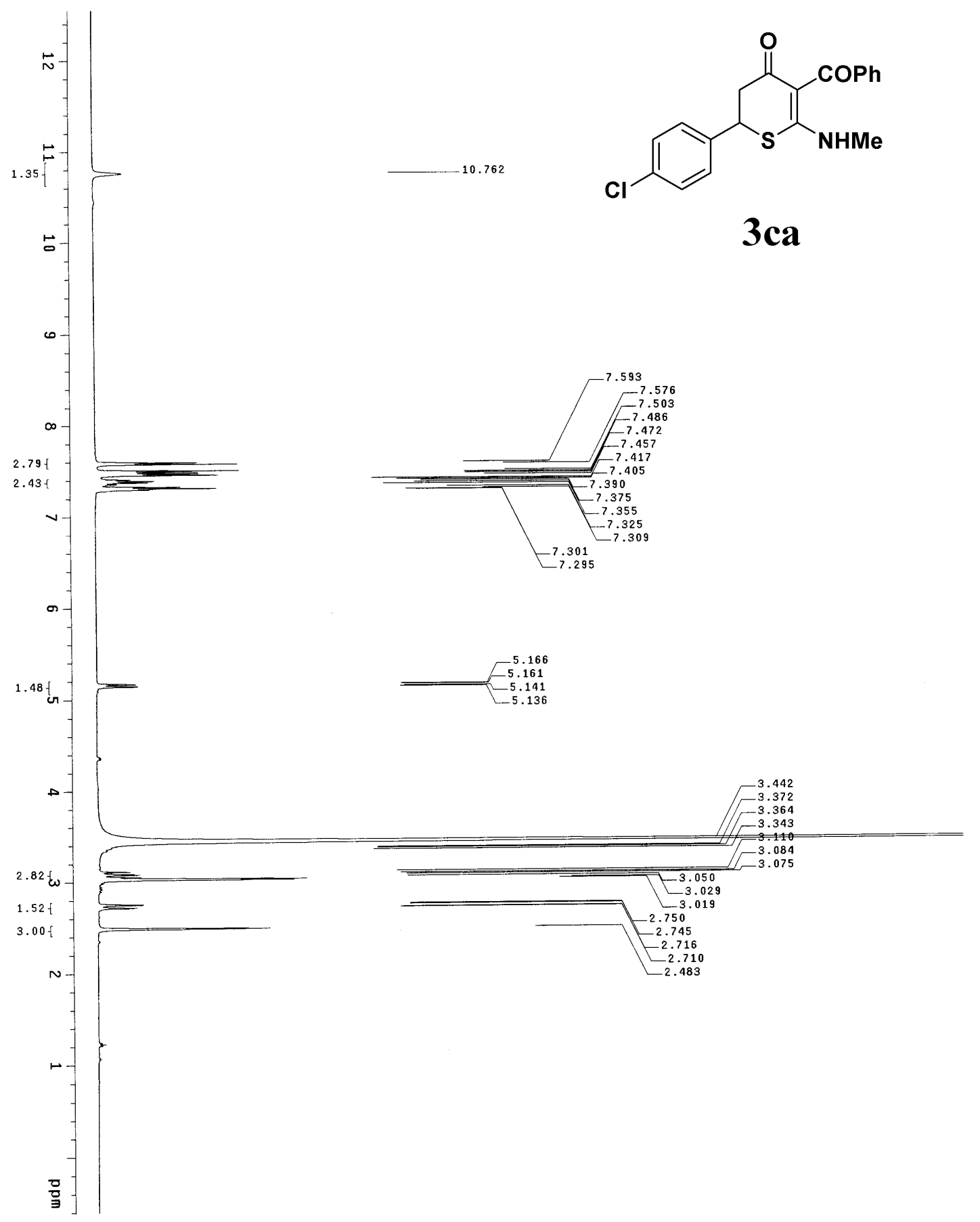




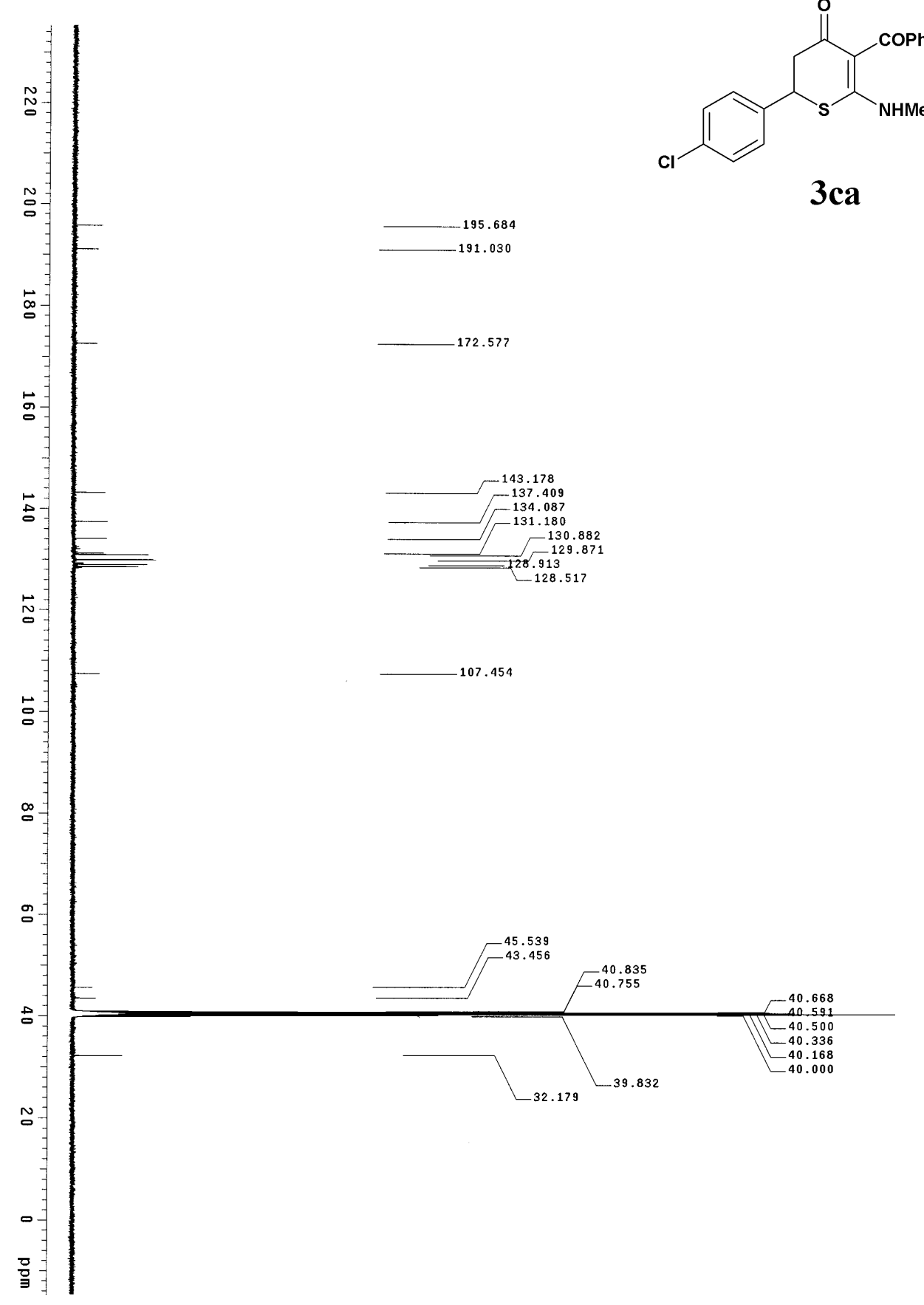




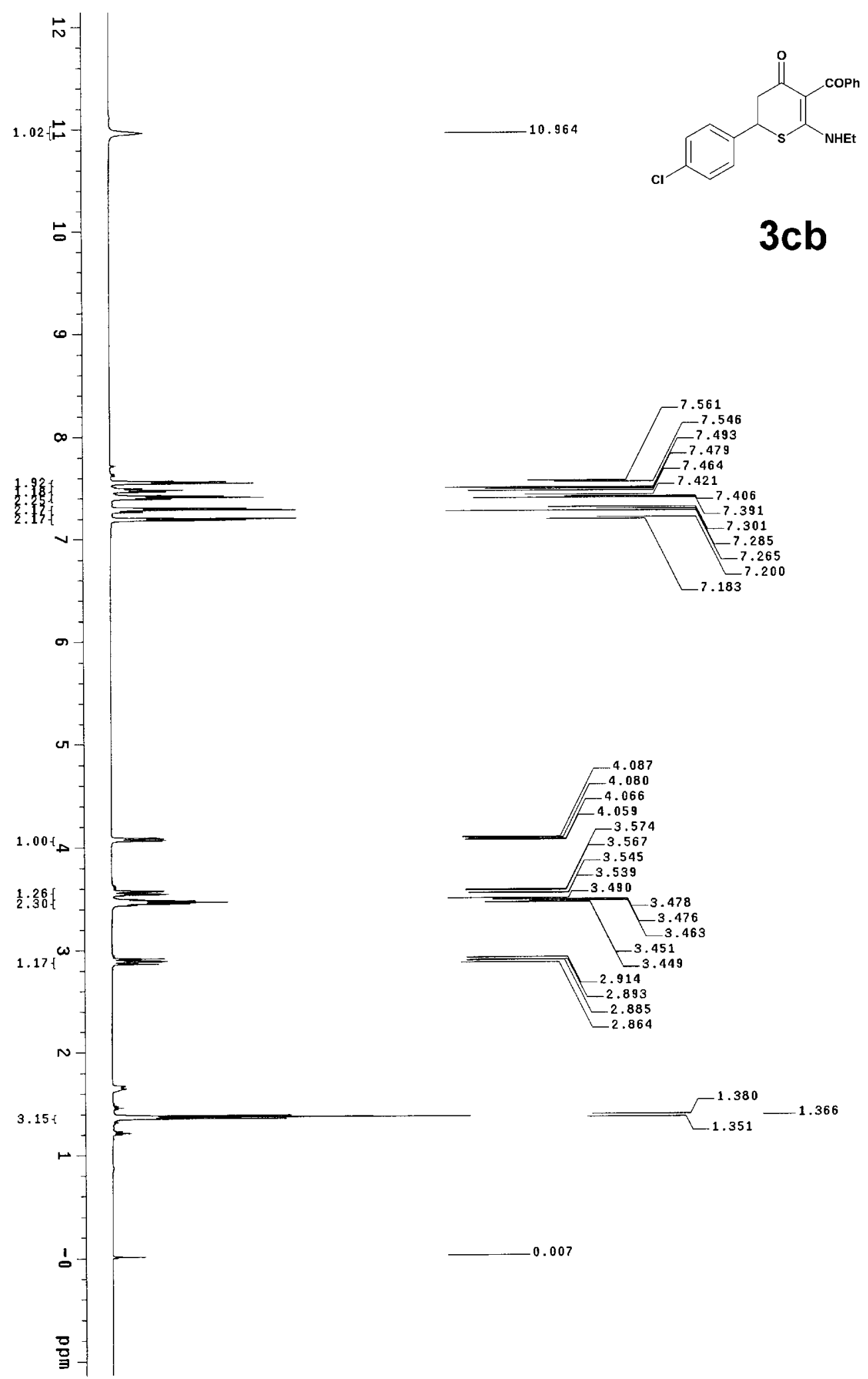




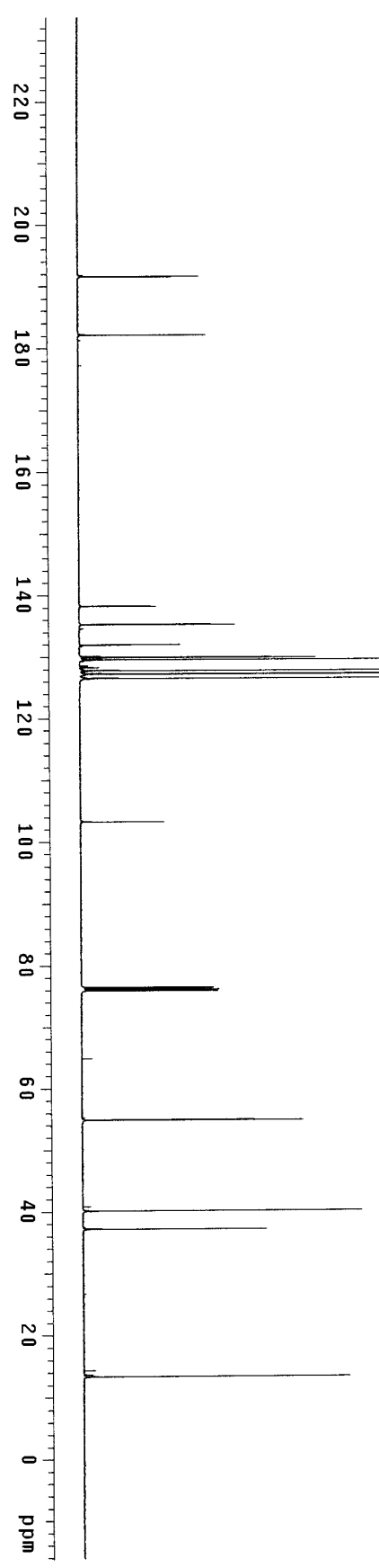<smiles>CCNC1=C(C(=O)Oc2ccccc2)C(=O)CC(c2ccc(Cl)cc2)S1</smiles>

$3 \mathrm{cb}$

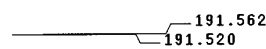

182.100

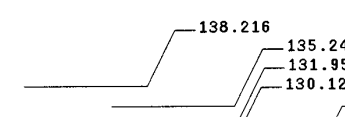

$\Gamma^{129.907}$

129.533
-128.293

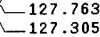

$-{ }^{126.4988}$

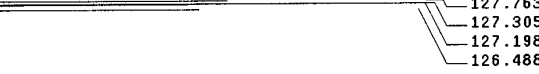

103.266
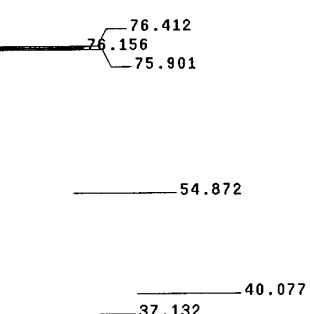

$-13.300$ 


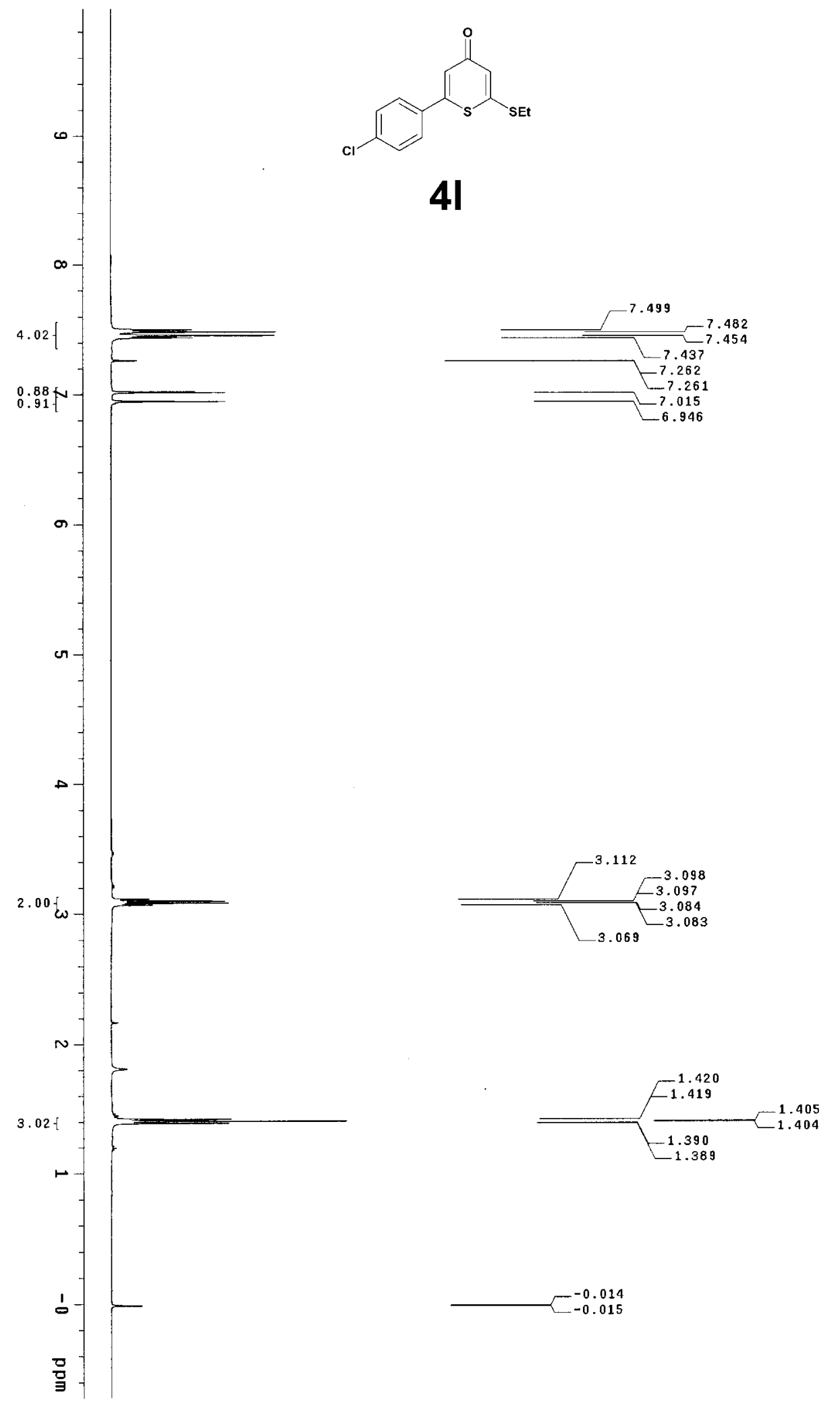




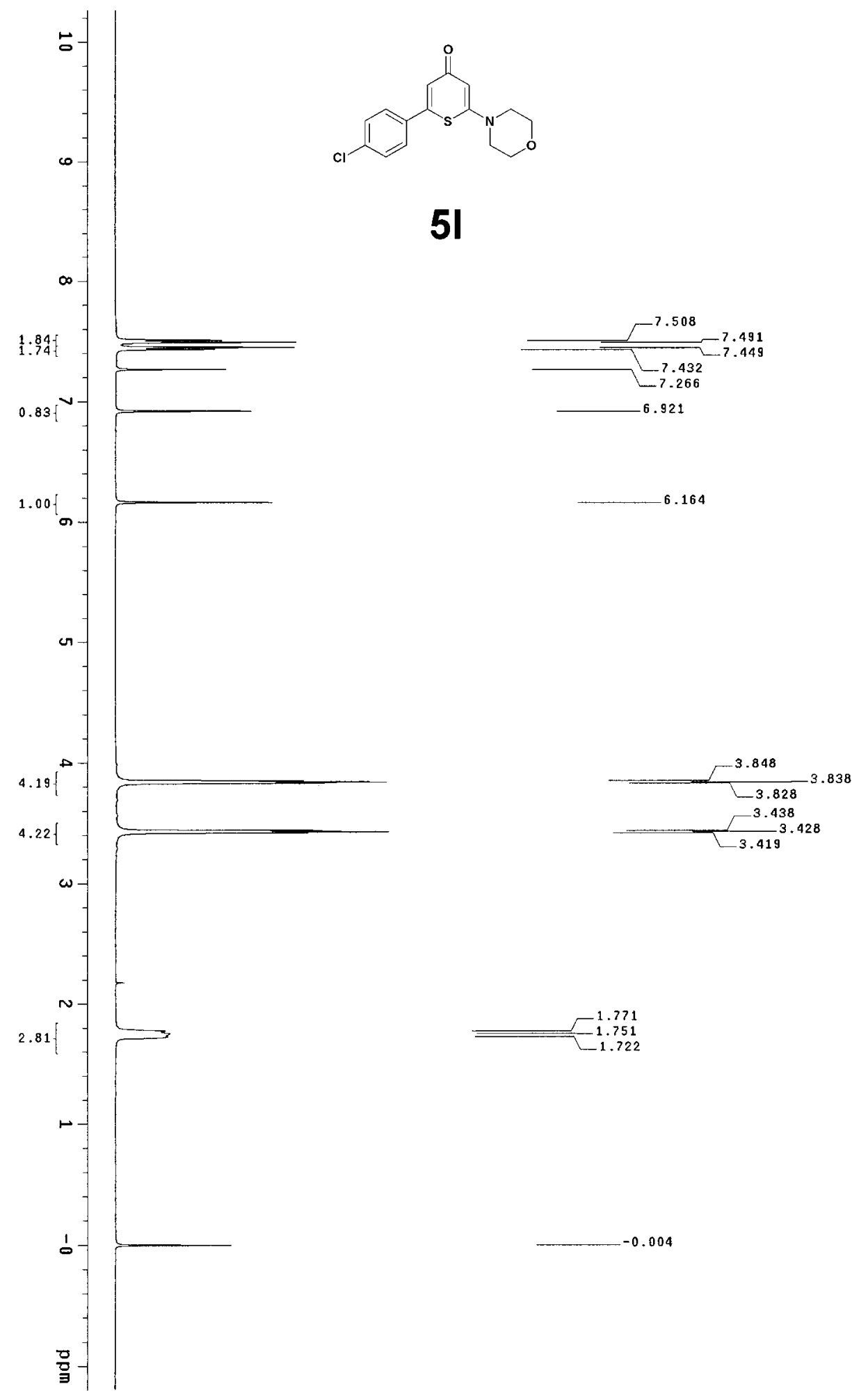

